\title{
An efficient multiple imputation algorithm for control-based and delta-adjusted pattern mixture models using SAS
}

\author{
Yongqiang Tang \\ Shire, 300 Shire Way, Lexington, MA 02421, USA*
}

July 8, 2016

\begin{abstract}
In clinical trials, mixed effects models for repeated measures (MMRM) and pattern mixture models (PMM) are often used to analyze longitudinal continuous outcomes. We describe a simple missing data imputation algorithm for the MMRM that can be easily implemented in standard statistical software packages such as SAS PROC MI. We explore the relationship of the missing data distribution in the control-based and delta-adjusted PMMs with that in the MMRM, and suggest an efficient imputation algorithm for these PMMs. The unobserved values in PMMs can be imputed by subtracting the mean difference in the posterior predictive distributions of missing data from the imputed values in MMRM. We also suggest a modification of the copy reference imputation procedure to avoid the possibility that after dropout, subjects from the active treatment arm will have better mean response trajectory than subjects who stay on the active treatment. The proposed methods are illustrated by the analysis of an antidepressant trial.
\end{abstract}

Keywords: Control-based imputation; Delta-adjusted imputation; Missing not at random; Mixed effects model for repeated measures

${ }^{*}$ to appear in Statistics in Biopharmaceutical research 


\section{Introduction}

Missing data are unavoidable in clinical trials, and can potentially result in biased treatment effect estimates. The primary analysis generally assumes a missing at random (MAR) mechanism. Suppose two subjects are identical (same treatment, same historical outcome) until a certain time point, and subject $A$ discontinues from the study after that time point, but subject $B$ remains on the treatment. The MAR mechanism implies that the future statistical behaviors of the two subjects are expected to be the same. This assumption may be unrealistic in some applications. For example, in a trial investigating a symptomatic treatment, the treatment benefit may disappear rapidly after discontinuation of the treatment, and dropouts and completers are unlikely to have the same statistical behaviors.

Recent regulatory guidelines (ICH E9, 1999; CHMP, 2010; National Research Council, 2010) emphasize the importance of sensitivity analyses to assess the robustness of the trial result under the missing not at random (MNAR) assumption. The pattern-mixture models (PMM) have become increasingly popular in sensitivity analyses for handling longitudinal clinical data with nonignorable missingness because the underlying missing data assumptions are easy to understand and interpret (Lu, 2014a). Two types of PMMs commonly used as MNAR sensitivity analyses in confirmatory trials are the control-based and deltaadjusted PMMs (Little and Yau, 1996; Carpenter et al., 2013; Ratitch et al., 2013). These PMMs provide de facto (effectiveness) estimands of the treatment effect, which measure the effect of the drug as actually taken using all randomized subjects under the intent to treat (ITT) principle (Carpenter et al., 2013; Ayele et al., 2014). Recently an addendum to ICH E9 was proposed relating to estimands and sensitivity analyses, and an expert group meeting sponsored by Statisticians in the Pharmaceutical Industry (PSI) was devoted to this topic prior to the release of the addendum (Phillips et al., 2016). The estimands from PMMs and MMRM correspond respectively to estimands 2 and 3 illustrated at the meeting (Phillips et al., 2016). These PMMs assume that the benefit among subjects in the active arm disappears or diminishes after treatment discontinuation, and therefore generally yield more conservative treatment effect estimates than the MMRM.

PMMs are often implemented via multiple imputation (MI). The simplest imputation algorithm is the sequential regression imputation (SRI) for monotone data, i.e. no miss- 
ing data prior to dropout (Little and Yau, 1996; Ratitch et al., 2013; Lu, 2014a). Tang (2015, 2016) proposed Markov chain Monte Carlo (MCMC) algorithms via monotone data augmentation (MDA) for missing data imputations in mixed effects models for repeated measures (MMRM) and a class of PMMs that assume the same observed data distribution as the MMRM. The MDA algorithm is a collapsed Gibbs sampler (Liu, 1994), in which the missing data $\boldsymbol{y}_{i \mathrm{~m}_{2}}$ 's after dropout are integrated out from the posterior distribution, and only the intermittent missing data $\boldsymbol{y}_{\mathrm{m}_{1}}$ are imputed in the imputation I-step. Compared to a full data augmentation (FDA) algorithm that imputes both $\boldsymbol{y}_{i_{\mathrm{m}}}$ 's and $\boldsymbol{y}_{i_{2}}$ 's in the I-step, the MDA algorithm generally converges to the same stationary distribution faster with smaller autocorrelation between posterior samples (Schafer, 1997; Tang, 2016).

The purpose of the paper is to propose a method that enables readers to write simple and efficient computer code for missing data imputations in the MMRM, control-based PMMs and delta-adjusted PMMs by building on existing software packages (e.g. PROC MI in SAS, Norm package in $\mathrm{R}$ ) that provide missing data imputation for incomplete multivariate normal data. The proposed method will produce the same posterior predictive distribution for the missing data as Tang (2015, 2016) MDA algorithm.

Our algorithm involves four steps, and details will be explained later in this section.

1. Construct a prior $p\left(\boldsymbol{\phi}_{x}, \boldsymbol{\phi}_{y}\right)=p\left(\boldsymbol{\phi}_{x}\right) p\left(\boldsymbol{\phi}_{y}\right)$ that can easily be specified in the software, where $\phi_{x}$ and $\phi_{y}$ are parameters associated respectively with the distributions of the covariates $\tilde{\boldsymbol{x}}_{i}$ and outcomes $\boldsymbol{y}_{i}$.

2. Impute missing data for MMRM (it assumes MAR) under the prior $p\left(\boldsymbol{\phi}_{x}, \boldsymbol{\phi}_{y}\right)$ using the software by pretending $\boldsymbol{w}_{i}=\left(\tilde{\boldsymbol{x}}_{i}^{\prime}, \boldsymbol{y}_{i}^{\prime}\right)^{\prime}$ is normally distributed.

3. Calculate the difference in the posterior mean of the missing data in MMRM and PMMs. It is a function of the posterior sample of $\phi_{y}$.

4. Subtracting the difference from the MAR-based imputation yields the imputed values for PMMs.

Section 2 describes the imputation algorithm for MMRM (i.e. steps 1 and 2). Let $f\left(\boldsymbol{y}_{i} \mid \tilde{\boldsymbol{x}}_{i}, \boldsymbol{\phi}_{y}\right)$ and $f\left(\boldsymbol{y}_{i o} \mid \tilde{\boldsymbol{x}}_{i}, \boldsymbol{\phi}_{y}\right)$ denote respectively the conditional distributions of the complete outcome $\boldsymbol{y}_{i}$ and observed outcome $\boldsymbol{y}_{i o}$ given the covariates $\tilde{\boldsymbol{x}}_{i}$ in the MMRM, and $p\left(\boldsymbol{\phi}_{y}\right)$ 
the prior. The missing values in the MMRM are imputed by pretending $\boldsymbol{w}_{i}=\left(\tilde{\boldsymbol{x}}_{i}^{\prime}, \boldsymbol{y}_{i}^{\prime}\right)^{\prime}$ follows a joint multivariate normal distribution with density $f\left(\boldsymbol{w}_{i} \mid \boldsymbol{\phi}_{x}, \boldsymbol{\phi}_{y}\right)=f\left(\boldsymbol{y}_{i} \mid \tilde{\boldsymbol{x}}_{i}, \boldsymbol{\phi}_{y}\right) f\left(\tilde{\boldsymbol{x}}_{i} \mid \boldsymbol{\phi}_{x}\right)$. But it does not require $\tilde{\boldsymbol{x}}_{i}$ to be normally distributed or continuous (that is, $f\left(\tilde{\boldsymbol{x}}_{i} \mid \boldsymbol{\phi}_{x}\right)$ is not the true density for $\tilde{\boldsymbol{x}}_{i}$ ). Since $\boldsymbol{\phi}_{x}$ and $\boldsymbol{\phi}_{y}$ have independent prior distributions, the marginal distribution of the posterior samples $\phi_{y}$ in this new algorithm will converge to its target distribution $f\left(\phi_{y} \mid\left(\tilde{\boldsymbol{x}}_{i}, \boldsymbol{y}_{i o}\right)^{\prime} s\right) \propto p\left(\boldsymbol{\phi}_{y}\right) \prod_{i} f\left(\boldsymbol{y}_{i o} \mid \tilde{\boldsymbol{x}}_{i}, \boldsymbol{\phi}_{y}\right)$.

Section 3 describes the imputation algorithm for control-based and delta-adjusted PMMs (i.e. steps 3 and 4). These PMMs assume the same observed data distribution as the MMRM. Therefore the posterior distributions of $\phi_{y}$ in these PMMs are the same as that in MMRM (Tang, 2016). Furthermore, the missing data after dropout are assumed to be normally distributed with the same covariance matrix as that in MMRM. Thus the missing values in PMMs can be imputed by subtracting the mean difference from the imputed values in the MMRM.

In the copy reference (CR) PMM, it is possible that the mean response after dropout among drug-treated subjects could be better than that among subjects who remain on the active treatment. We propose a modification of the CR procedure in Section 3.

Section 4 illustrates the proposed methods by the analysis of an antidepressant trial. Section 5 will compare the proposed imputation algorithm with some existing methods.

\section{MCMC algorithms for MMRM}

\subsection{MMRM and related MCMC algorithms}

We consider a two-arm trial, where $n$ subjects are randomly assigned to the active $\left(g_{i}=1\right)$ or control $\left(g_{i}=0\right)$ treatment. Let $\boldsymbol{y}_{i}=\left(y_{i 1}, \ldots, y_{i p}\right)^{\prime}$ denote the outcomes at the $p$ postbaseline visits, and $\left(x_{i 1}, \ldots, x_{i d}\right)^{\prime}$ the baseline covariates for subject $i$. We assume that $x_{i k}$ 's are fully observed. In general, $\boldsymbol{y}_{i}$ 's will be only partially observed. Let $r_{i}$ index the dropout pattern for subject $i$ according to the last observation. A subject is in pattern $r_{i}=s$ if $s$ is last visit that the subject has a measurement observed, and $r_{i}=0$ if a subject has no post-baseline assessment. Without loss of generality, we sort the data so that subjects in pattern $s$ are arranged before subjects in pattern $t$ if $s>t$. Suppose after filling in the 
intermittent missing data, $y_{i k}$ is not missing in the first $n_{k}$ subjects.

Let $\boldsymbol{y}_{i o}, \boldsymbol{y}_{\mathrm{im}_{1}}$ and $\boldsymbol{y}_{\mathrm{im}_{2}}$ denote respectively the observed data, intermittent missing data, and missing data after dropout for subject $i$. Let $\overrightarrow{\boldsymbol{y}}_{i t}=\left(y_{i 1}, \ldots, y_{i t}\right)$. Then $\boldsymbol{y}_{i o}\left(\boldsymbol{y}_{i m_{1}}\right)$ is the observed (missing) part of $\overrightarrow{\boldsymbol{y}}_{i r_{i}}$. Let $x_{i 0} \equiv 1, \tilde{\boldsymbol{x}}_{i}=\left(x_{i 1}, \ldots, x_{i d}, g_{i}\right)^{\prime}$ and $\boldsymbol{x}_{i}=$ $\left(x_{i 0}, \ldots, x_{i d}, g_{i}\right)^{\prime}$. Let $Y_{o}, Y_{\mathrm{m}_{1}}, Y_{\mathrm{m}_{2}}$, and $X$ denote respectively the observed outcomes, intermittent missing data, missing data after dropout, and covariates from all subjects.

The following MMRM is often used as the primary analysis for longitudinal outcomes collected at a fixed number of time points (Siddiqui et al., 2009). It assumes MAR.

$$
\boldsymbol{y}_{i}=\left(y_{i 1}, \ldots, y_{i p}\right)^{\prime} \sim N\left[\left(\boldsymbol{\alpha}_{1}^{\prime} \boldsymbol{x}_{i}, \ldots, \boldsymbol{\alpha}_{p}^{\prime} \boldsymbol{x}_{i}\right)^{\prime}, \Sigma\right]
$$

where $q=d+2$, and $\boldsymbol{\alpha}_{j}=\left(\alpha_{j 0}, \ldots, \alpha_{j d}, \delta_{j}\right)^{\prime}$ be a $q \times 1$ vector of the covariate and treatment effects at visit $j$. The model includes an unstructured treatment $\times$ visit interaction effect, and thus allows the direct estimate of the treatment effect at each scheduled visit (Siddiqui et al., 2009). The within subject errors are modeled by an unstructured covariance.

Let $\Sigma=U^{-1} \Lambda\left(U^{\prime}\right)^{-1}$ be the LDL decomposition of $\Sigma$, where $\Lambda=\operatorname{diag}\left(\gamma_{1}^{-1}, \ldots, \gamma_{p}^{-1}\right)$, and $U=\left[\begin{array}{cccc}1 & 0 & \ldots & 0 \\ -\beta_{21} & 1 & \ldots & 0 \\ & \ldots & \ldots & 0 \\ -\beta_{p 1} & \ldots & -\beta_{p, p-1} & 1\end{array}\right]$. Then (1) can be written as $U \boldsymbol{y}_{i} \sim N\left[\left(\underline{\boldsymbol{\alpha}}_{1}^{\prime} \boldsymbol{x}_{i}, \ldots, \underline{\boldsymbol{\alpha}}_{p}^{\prime} \boldsymbol{x}_{i}\right)^{\prime}, \Lambda\right]$, or equivalently as the product of the following regression models

$$
y_{i j} \mid \overrightarrow{\boldsymbol{y}}_{i, j-1}, \boldsymbol{x}_{i} \sim N\left(\boldsymbol{\theta}_{j}^{\prime} \boldsymbol{z}_{i, j-1}, \gamma_{j}^{-1}\right) \text { for } j \leq p,
$$

where $\underline{\boldsymbol{\alpha}}_{j}=\left(\underline{\alpha}_{j 0}, \ldots, \underline{\alpha}_{j d}, \underline{\delta}_{j}\right)^{\prime}=\boldsymbol{\alpha}_{j}-\sum_{t=1}^{j-1} \beta_{j t} \boldsymbol{\alpha}_{t}, \boldsymbol{\beta}_{j}=\left(\beta_{j 1}, \ldots, \beta_{j j-1}\right)^{\prime}, \boldsymbol{\theta}_{j}=\left(\underline{\boldsymbol{\alpha}}_{j}^{\prime}, \boldsymbol{\beta}_{j}^{\prime}\right)^{\prime}$, and $\boldsymbol{z}_{i j}=\left(\boldsymbol{x}_{i}^{\prime}, \overrightarrow{\boldsymbol{y}}_{i j}^{\prime}\right)^{\prime}$. Let $\boldsymbol{\alpha}=\left(\boldsymbol{\alpha}_{1}, \ldots, \boldsymbol{\alpha}_{p}\right)^{\prime}$.

Tang (2015) considered a conjugate normal-inverse-Wishart (NIW) prior for $(\Sigma, \boldsymbol{\alpha})$. 
That is, $\Sigma \sim \mathcal{W}^{-1}\left(A, \nu_{0}\right), \operatorname{vec}(\boldsymbol{\alpha}) \mid \Sigma \sim N\left(\operatorname{vec}\left(\boldsymbol{\alpha}_{0}\right), M^{+} \otimes \Sigma\right)$, and the prior density is

$$
\begin{aligned}
p(\boldsymbol{\alpha}, \Sigma) \propto p(\Sigma) p(\boldsymbol{\alpha} \mid \Sigma) & \propto\left\{|\Sigma|^{-\frac{\nu_{0}+p+1}{2}} \exp \left[-\frac{1}{2} \operatorname{tr}\left(A \Sigma^{-1}\right)\right]\right\} \\
& \left\{|\Sigma|^{-\frac{r}{2}} \exp \left[-\frac{1}{2}\left(\operatorname{vec}\left(\boldsymbol{\alpha}-\boldsymbol{\alpha}_{0}\right)\right)^{\prime}\left(M \otimes \Sigma^{-1}\right)\left(\operatorname{vec}\left(\boldsymbol{\alpha}-\boldsymbol{\alpha}_{0}\right)\right)\right]\right\},
\end{aligned}
$$

where $\mathcal{W}^{-1}\left(A, \nu_{0}\right)$ denotes an inverse-Wishart distribution with $\nu_{0}$ degrees of freedom, and $p \times p$ scale matrix $A, \boldsymbol{\alpha}_{0}$ is a $p \times q$ fixed matrix containing the prior mean of $\boldsymbol{\alpha}$, and $M^{+}$ is Moore-Penrose inverse of a $q \times q$ fixed matrix $M=\left(m_{i j}\right)$ with rank $r$. The prior for $q-r$ covariates is flat. If a covariate has a flat prior, the corresponding mean components in $\boldsymbol{\alpha}_{0}$ and (co)variance elements in $M$ will be set to 0 . For example, suppose historical information is available only on the placebo response, and there is no baseline covariate $(d=0)$. We may put a (weakly) informative prior on the intercept, and a flat prior on the

treatment effect. Then $M=\left[\begin{array}{cc}m_{11} & 0 \\ 0 & 0\end{array}\right]$ and $\boldsymbol{\alpha}_{0}=\left(\boldsymbol{\alpha}_{01}, \mathbf{0}_{p}\right)$, where $\mathbf{0}_{k}$ is a $k \times 1$ vector of zeros. If the prior is flat on all covariates $(r=0)$, then $M=\mathbf{0}$. We need to pre-specify $A, \nu_{0}, \boldsymbol{\alpha}_{0}$ and $M$ in the prior. For Jeffrey's prior $p(\boldsymbol{\alpha}, \Sigma) \propto|\Sigma|^{-(p+1) / 2}$, we have $\nu_{0}=0$, $A=\mathbf{0}, \boldsymbol{\alpha}_{0}=\mathbf{0}$, and $M=\mathbf{0}$. We do not require $A$ to be positive definite.

Tang (2015, 2016) developed the MDA (called MDA-T) and FDA (called FDA-T) algorithms for MMRM. They rely on the fact that $\left(\boldsymbol{\theta}_{j}, \gamma_{j}\right)$ 's are independent in the posterior distribution given the augmented monotone $\left(Y_{o}, Y_{\mathrm{m} 1}\right)$ or complete $\left(Y_{o}, Y_{\mathrm{m} 1}, Y_{\mathrm{m} 2}\right)$ data since the corresponding likelihood can be factored as the product of $p$ independent likelihoods for $\left(\boldsymbol{\theta}_{j}, \gamma_{j}\right)$ 's based on (2), and the prior for $(\boldsymbol{\alpha}, \Sigma)$ can be factored into independent densities for $\left(\boldsymbol{\theta}_{j}, \gamma_{j}\right)$ 's. The MDA algorithm is generally recommended in real applications because it converges to the same stationary distribution faster than the FDA algorithm (Schafer, 1997; Tang, 2016). Appendix A.1 provides a brief review of the two algorithms.

\subsection{MCMC algorithm for MMRM using SAS PROC MI}

This section describes a simple method to implement Bayesian MMRM analysis in a software package that can generate multiple imputations for incomplete multivariate normal data. We illustrate the method using SAS PROC MI since SAS is more commonly used 
to analyze clinical trials in the pharmaceutical industry. The underlying idea is to run a Bayesian analysis for $\boldsymbol{w}_{i}=\left(\tilde{\boldsymbol{x}}_{i}^{\prime}, \boldsymbol{y}_{i}^{\prime}\right)^{\prime}$ by pretending $\boldsymbol{w}_{i}$ follows a multivariate normal distribution $\boldsymbol{w}_{i} \stackrel{i . i . d}{\sim} N\left(\boldsymbol{\alpha}_{w}, \Sigma_{w}\right)$, where $\boldsymbol{\alpha}_{w}=\left[\begin{array}{l}\boldsymbol{\alpha}_{x} \\ \boldsymbol{\alpha}_{y}\end{array}\right]$ and $\Sigma_{w}=\left[\begin{array}{cc}\Sigma_{x x} & \Sigma_{y x}^{\prime} \\ \Sigma_{y x} & \Sigma_{y y}\end{array}\right]$. The likelihood for $\left(\boldsymbol{\alpha}_{w}, \Sigma_{w}\right)$ can be decomposed as independent likelihoods for $\left(\boldsymbol{\alpha}_{x}, \Sigma_{x x}\right)$ and $(\boldsymbol{\alpha}, \Sigma)$,

$$
\prod_{i=1}^{n} f\left(\boldsymbol{w}_{i} ; \boldsymbol{\alpha}_{w}, \Sigma_{w}\right)=\left\{\prod_{i=1}^{n} f\left(\tilde{\boldsymbol{x}}_{i} ; \boldsymbol{\alpha}_{x}, \Sigma_{x x}\right)\right\}\left\{\prod_{i=1}^{n} f\left(\boldsymbol{y}_{i} \mid \boldsymbol{x}_{i} ; \boldsymbol{\alpha}, \Sigma\right)\right\}
$$

The method yields the same posterior distribution of $(\boldsymbol{\alpha}, \Sigma)$ as any valid MCMC algorithm for (11) if the prior for $\left(\boldsymbol{\alpha}_{w}, \Sigma_{w}\right)$ can be factored into independent densities as $p\left(\boldsymbol{\alpha}_{w}, \Sigma_{w}\right)=$ $p\left(\boldsymbol{\alpha}_{x}, \Sigma_{x x}\right) p(\boldsymbol{\alpha}, \Sigma)$, where $p(\boldsymbol{\alpha}, \Sigma)$ is defined in (3). We assume $\tilde{\boldsymbol{x}}_{i}$ 's are fully observed, but $\tilde{\boldsymbol{x}}_{i}$ may not be normally distributed.

For missing data imputation, it is convenient to reparameterize $\left(\boldsymbol{\alpha}_{w}, \Sigma_{w}\right)$ based on the LDL decomposition of $\Sigma_{w}=U_{w}^{-1} \Lambda_{w}\left(U_{w}^{\prime}\right)^{-1}$, where $U_{w}$ is a lower triangular matrix with all 1's on the diagonal, and $\Lambda_{w}=\operatorname{diag}\left(\gamma_{1}^{*^{-1}}, \ldots, \gamma_{q-1+p}^{*^{-1}}\right)$. Let $-\boldsymbol{\beta}_{j}^{*}$ denote the first $j-1$ elements of row $j$ in $U_{w}, \underline{\alpha}_{j}^{*}$ the $j$-th element of $\underline{\boldsymbol{\alpha}}_{w}=U_{w} \boldsymbol{\alpha}_{w}$, and $\boldsymbol{\theta}_{j}^{*}=\left(\underline{\alpha}_{j}^{*}, \boldsymbol{\beta}_{j}^{*}\right)^{\prime}$. Note that $\left(\boldsymbol{\theta}_{j+q-1}^{*}, \gamma_{j+q-1}^{*}\right)$ has the same interpretation as $\left(\boldsymbol{\theta}_{j}, \gamma_{j}\right)$ defined in (2). Then $\left(\boldsymbol{\alpha}_{x}, \Sigma_{x x}\right)$ can be expressed a function of $\boldsymbol{\phi}_{x}=\left\{\left(\boldsymbol{\theta}_{j}^{*}, \gamma_{j}^{*}\right): j=1, \ldots, q-1\right\}$, and $(\boldsymbol{\alpha}, \Sigma)$ is a function of $\boldsymbol{\phi}_{y}=\left\{\left(\boldsymbol{\theta}_{j}^{*}, \gamma_{j}^{*}\right): j=q, \ldots, q+p-1\right\}=\left\{\left(\boldsymbol{\theta}_{j}, \gamma_{j}\right): j=1, \ldots, p\right\}$.

Lemma 1 below provides the theoretical basis for the proposed algorithm, in which a NIW prior is constructed for $\left(\boldsymbol{\alpha}_{w}, \Sigma_{w}\right)$. Its proof will be deferred to Appendix A.2. One needs to create the following quantities from $A, \nu_{0}, \boldsymbol{\alpha}_{0}$ and $M$ to define the NIW prior.

- $m_{11}$ is $(1,1)$ entry of $M=\left[\begin{array}{ll}m_{11} & M_{12} \\ M_{21} & M_{22}\end{array}\right]$. Note that $m_{11}^{+}=m_{11}^{-1}$ if $m_{11}>0$, and $m_{11}^{+}=0$ if $m_{11}=0$.

- $A_{w}=\left[\begin{array}{cc}M^{*} & M^{*} \boldsymbol{\alpha}_{0}^{* \prime} \\ \boldsymbol{\alpha}_{0}^{*} M^{*} & A+\boldsymbol{\alpha}_{0}^{*} M^{*} \boldsymbol{\alpha}_{0}^{* \prime}\end{array}\right]$, where $\boldsymbol{\alpha}_{0}^{*}$ is a $p \times(q-1)$ matrix containing the last $q-1$ columns of $\boldsymbol{\alpha}_{0}, M^{*}=M_{22}$ if $m_{11}=0$, and $M^{*}=M_{22}-M_{21} M_{12} / m_{11}$ if $m_{11}>0$.

- $\nu_{w}=\nu_{0}+r-(q-1)$ if $m_{11}=0$, and $\nu_{w}=\nu_{0}+r-q$ if $m_{11} \neq 0$. 
- $\boldsymbol{\alpha}_{w 0}=\mathbf{0}_{q-1+p}$ if $m_{11}=0$, and $\boldsymbol{\alpha}_{w 0}=m_{11}^{-1}\left[\begin{array}{c}M_{21} \\ M_{1}^{*}\end{array}\right]$ if $m_{11} \neq 0$, where $M_{1}^{*}=\boldsymbol{\alpha}_{0}\left[\begin{array}{l}m_{11} \\ M_{21}\end{array}\right]$.

In large confirmatory trials, a flat prior is generally put on $\boldsymbol{\alpha}(r=0, M=\mathbf{0})$, then we have $A_{w}=\left[\begin{array}{ll}\mathbf{0} & \mathbf{0} \\ \mathbf{0} & A\end{array}\right], \nu_{w}=\nu_{0}-q+1, m_{11}=0$ and $\boldsymbol{\alpha}_{w 0}=\mathbf{0}_{q-1+p}$.

Lemma 1 Suppose in a Bayesian analysis, $\left(\boldsymbol{\alpha}_{w}, \Sigma_{w}\right)$ or $\left(\underline{\boldsymbol{\alpha}}_{w}, \Lambda_{w}\right)$ are sampled under the $N I W$ prior $\Sigma_{w} \sim \mathcal{W}^{-1}\left(A_{w}, \nu_{w}\right)$ and $\boldsymbol{\alpha}_{w} \mid \Sigma_{w} \sim N\left(\boldsymbol{\alpha}_{w 0}, m_{11}^{+} \Sigma_{w}\right)$ (when $m_{11}=0, f\left(\boldsymbol{\alpha}_{w} \mid \Sigma_{w}\right) \propto$ constant) by pretending $\boldsymbol{w}_{i} \stackrel{i . i . d}{\sim} N\left(\boldsymbol{\alpha}_{w}, \Sigma_{w}\right)$. Then

(a) $\left(\boldsymbol{\theta}_{j}^{*}, \gamma_{j}^{*}\right)$ 's are independent in the prior, and $\left(\boldsymbol{\theta}_{j+q-1}^{*}, \gamma_{j+q-1}^{*}\right)$ has the same prior as $\left(\boldsymbol{\theta}_{j}, \gamma_{j}\right)$ defined in (17) in Appendix A.1.

(b) $\left(\boldsymbol{\alpha}_{x}, \Sigma_{x x}\right)$ and $(\boldsymbol{\alpha}, \Sigma)$ are independent in the prior.

(c) If (1) holds, but $\tilde{\boldsymbol{x}}_{i}$ may not be normally distributed, the posterior distributions of $\boldsymbol{\phi}_{y}$ and $(\boldsymbol{\alpha}, \Sigma)$ are the same as as that from the MCMC algorithms discussed in Section 2.1.

In SAS, MCMC sampling for incomplete multivariate normal data can be conveniently performed using PROC MI. It is flexible to specify priors in SAS. The prior on $\boldsymbol{\alpha}_{w}$ could be flat (i.e. $m_{11}=0, f\left(\boldsymbol{\alpha}_{w} \mid \Sigma_{w}\right) \propto$ constant), and $A_{w}$ may not be of full rank. SAS PROC MI produces posterior samples of $\left(\boldsymbol{\alpha}_{w}, \Sigma_{w}\right)$. One can recreate $\boldsymbol{\phi}_{y}=\left\{\left(\boldsymbol{\theta}_{j}, \gamma_{j}\right): j=1, \ldots, p\right\}$ from $\left(\boldsymbol{\alpha}_{w}, \Sigma_{w}\right)$ based on LDL decomposition of $\Sigma_{w}$, and recreate $(\boldsymbol{\alpha}, \Sigma)$ from $\left(\boldsymbol{\theta}_{j}, \gamma_{j}\right)$ 's based on $\Sigma=U^{-1} \Lambda\left(U^{\prime}\right)^{-1}$, and $\boldsymbol{\alpha}=U^{-1}\left[\underline{\boldsymbol{\alpha}}_{1}, \ldots, \underline{\boldsymbol{\alpha}}_{p}\right]^{\prime}$.

SAS PROC MI implements Schafer (1997) MDA algorithm (called MDA-SAS) under Jeffrey's prior, and a FDA algorithm (called FDA-SAS) under a general prior. To provide further insight into the proposed method, we will explain in Appendix A.3 why the two algorithms in SAS yield the same posterior distribution of $\phi_{y}$ as the MDA-T and FDA-T algorithms without the use of Lemma 1.

\section{Missing data imputation in PMMs}

PMMs are generally implemented via MI. In MI, $m(m>1)$ complete datasets are imputed, and analyzed using a standard method (e.g. MMRM, analysis of covariance model (ANCOVA)). The results from the $m$ complete datasets are then combined using Rubin 
(1987) rule. One major challenge in the MI inference is the imputation of missing data. We will introduce a simple and efficient strategy for missing data imputation in a class of PMMs that assume the same observed data distribution as MMRM.

The joint distribution of $\left(\boldsymbol{y}_{i}, r_{i}\right)$ in PMMs can be factored as

$$
\begin{aligned}
f\left(\boldsymbol{y}_{i}, r_{i} \mid \boldsymbol{\phi}, \boldsymbol{\pi}, \boldsymbol{x}_{i}\right) & =f\left(\boldsymbol{y}_{i} \mid r_{i}, \phi_{y}, \boldsymbol{x}_{i}\right) f\left(r_{i} \mid \boldsymbol{\pi}, \boldsymbol{x}_{i}\right) \\
& =f\left(\overrightarrow{\boldsymbol{y}}_{i r_{i}} \mid \boldsymbol{\phi}_{y}, \boldsymbol{x}_{i}\right) Q\left(\boldsymbol{y}_{i \mathrm{~m}_{2}} \mid \overrightarrow{\boldsymbol{y}}_{i r_{i}}, \boldsymbol{\phi}_{y}, \boldsymbol{x}_{i}\right) f\left(r_{i} \mid \boldsymbol{\pi}, \boldsymbol{x}_{i}\right),
\end{aligned}
$$

where $f\left(r_{i} \mid \boldsymbol{\pi}, \boldsymbol{x}_{i}\right)$ models the marginal proportions of subjects in each pattern, and $Q\left(\boldsymbol{y}_{i \mathrm{~m}_{2}} \mid \overrightarrow{\boldsymbol{y}}_{i r_{i}}, \boldsymbol{x}_{i}, \boldsymbol{\phi}_{y}\right)$ is the conditional distribution of $\boldsymbol{y}_{i \mathrm{~m}_{2}}$ given $\overrightarrow{\boldsymbol{y}}_{i r_{i}}=\left(\boldsymbol{y}_{i o}^{\prime}, \boldsymbol{y}_{i \mathrm{~m}_{1}}^{\prime}\right)^{\prime}$ and $\boldsymbol{x}_{i}$. A common feature of these PMMs is that the outcome $\overrightarrow{\boldsymbol{y}}_{i r_{i}}$ before dropout has the same marginal distribution as that defined in (1). That is, the observed data $\left(\boldsymbol{y}_{i o}\right.$ 's) distributions are identical in PMMs and MMRM, and the intermittent missing data $\left(\boldsymbol{y}_{i_{1}}{ }^{\prime} s\right)$ are MAR.

In PMMs, the complete data likelihood can be written as

$$
\left\{\prod_{i=1}^{n} f\left(\overrightarrow{\boldsymbol{y}}_{i r_{i}} \mid \boldsymbol{x}_{i}, \boldsymbol{\phi}_{y}\right) \prod_{i=1}^{n} Q\left(\boldsymbol{y}_{i \mathrm{~m}_{2}} \mid \overrightarrow{\boldsymbol{y}}_{i r_{i}}, \boldsymbol{x}_{i}, \boldsymbol{\phi}_{y}\right)\right\}\left\{\prod_{i=1}^{n} f\left(r_{i} \mid \boldsymbol{x}_{i}, \boldsymbol{\pi}\right)\right\} .
$$

If the prior $\phi_{y} \sim p\left(\phi_{y}\right)$ is the same as specified in section 2, and an independent prior is put on $\boldsymbol{\pi}$, then $\boldsymbol{\pi}$ and $\left(\phi_{y}, Y_{\mathrm{m}_{1}}, Y_{\mathrm{m}_{2}}\right)$ are independent in the posterior distribution, and the joint posterior distribution of $\left(\phi_{y}, Y_{\mathrm{m}_{1}}, Y_{\mathrm{m}_{2}}\right)$ is given by

$$
\operatorname{pos}_{2}\left(\phi_{y}, Y_{\mathrm{m}_{1}}, Y_{\mathrm{m}_{2}} \mid Y_{o}, X\right) \propto \operatorname{pos}_{1}\left(\phi_{y}, Y_{\mathrm{m}_{1}} \mid Y_{o}, X\right) \prod_{i=1}^{n} Q\left(\boldsymbol{y}_{i \mathrm{~m}_{2}} \mid \overrightarrow{\boldsymbol{y}}_{i r_{i}}, \boldsymbol{x}_{i}, \boldsymbol{\phi}_{y}\right)
$$

where $\operatorname{pos}_{1}\left(\boldsymbol{\phi}_{y}, Y_{\mathrm{m}_{1}} \mid Y_{o}, X\right) \propto p\left(\boldsymbol{\phi}_{y}\right) \prod_{i=1}^{n} f\left(\overrightarrow{\boldsymbol{y}}_{i r_{i}} \mid \boldsymbol{x}_{i}, \boldsymbol{\phi}_{y}\right)$. In both MMRM and PMMs, the posterior distribution of $\left(\phi_{y}, Y_{\mathrm{m}_{1}}\right)$ is $\operatorname{pos}_{1}\left(\phi_{y}, Y_{\mathrm{m}_{1}} \mid Y_{o}, X\right)$.

We propose the following two-step procedure for imputing missing data from their posterior predictive distribution in PMMs

1. Sample $\left(\phi_{y}, Y_{\mathrm{m}_{1}}\right)$ from the marginal posterior distribution $\operatorname{pos}_{1}\left(\boldsymbol{\phi}_{y}, Y_{\mathrm{m}_{1}} \mid Y_{o}, X\right)$ using an algorithm described in Section 2;

2. Impute $\boldsymbol{y}_{i \mathrm{~m}_{2}}$ from $Q\left(\boldsymbol{y}_{i \mathrm{~m}_{2}} \mid \overrightarrow{\boldsymbol{y}}_{i_{i}}, \boldsymbol{x}_{i}, \boldsymbol{\phi}_{y}\right)$ for $i=1, \ldots, n$. 
Because step 1 of the algorithm does not depend on step 2, it is equivalent to running step 1 until convergence, and then sampling $\boldsymbol{y}_{i \mathrm{~m}_{2}}$ 's from $Q\left(\boldsymbol{y}_{i \mathrm{~m}_{2}} \mid \overrightarrow{\boldsymbol{y}}_{i r_{i}}, \boldsymbol{x}_{i}, \boldsymbol{\phi}_{y}\right)$. The proposed approach is not necessarily a MCMC algorithm. For example, we may run step 1 via a FDA approach under MAR, and then run step 2 to impute $\boldsymbol{y}_{i \mathrm{~m}_{2}}$ 's in PMMs. However, the joint distribution of $\left(\phi_{y}, Y_{\mathrm{m}_{1}}, Y_{\mathrm{m}_{2}}\right)$ will converge to the target distribution (4).

Suppose the joint distribution of $\boldsymbol{y}_{i}=\left(\overrightarrow{\boldsymbol{y}}_{i s}^{\prime}, \boldsymbol{y}_{i \mathrm{~m} 2}^{\prime}\right)^{\prime}$ is normal with mean $\left(\boldsymbol{\eta}_{i s}^{\prime}, \boldsymbol{\eta}_{\boldsymbol{i m}}^{\prime}\right)^{\prime}$ and covariance $\Sigma=\left[\begin{array}{cc}\Sigma_{11^{s}} & \Sigma_{21^{s}}^{\prime} \\ \Sigma_{21} & \Sigma_{22^{\circ}}\end{array}\right]$ in pattern $s$. The conditional distribution $Q\left(\boldsymbol{y}_{i \mathrm{~m}_{2}} \mid \overrightarrow{\boldsymbol{y}}_{i r_{i}}, \boldsymbol{x}_{i}, \boldsymbol{\phi}_{y}\right)$ of $\boldsymbol{y}_{i \mathrm{~m}_{2}}$ given $\overrightarrow{\boldsymbol{y}}_{i_{i}}$ is (Carpenter et al., 2013)

$$
\boldsymbol{y}_{i \mathrm{~m}_{2}} \mid \overrightarrow{\boldsymbol{y}}_{i s}, r_{i}=s \sim N\left[\boldsymbol{\eta}_{i m}+\Sigma_{21^{s}} \Sigma_{11^{s}}^{-1}\left(\overrightarrow{\boldsymbol{y}}_{i s}-\boldsymbol{\eta}_{i s}\right), \Sigma_{22^{s}}-\Sigma_{21^{s}} \Sigma_{11^{s}}^{-1} \Sigma_{21^{s}}^{\prime}\right]
$$

Because all models assume the same observed data distribution and a common covariance matrix $\operatorname{var}\left(\boldsymbol{y}_{i}\right)=\Sigma$ across patterns, the missing data distribution $Q\left(\boldsymbol{y}_{i \mathrm{~m}_{2}} \mid \overrightarrow{\boldsymbol{y}}_{i r_{i}}, \boldsymbol{x}_{i}, \boldsymbol{\phi}_{y}\right)$ in these models differs only in $\boldsymbol{\eta}_{i m}$. Therefore, the imputed values in PMMs can be obtained by subtracting the difference in mean $\boldsymbol{y}_{i \mathrm{~m}_{2}}$ from the imputed values in MMRM while the imputation for MMRM can be done using SAS PROC MI. All models assume MAR in the control arm, and hence have the same imputed values among control subjects.

For the purpose of missing data imputation, it is more convenient to express the conditional distribution $Q\left(\boldsymbol{y}_{i \mathrm{~m}_{2}} \mid \overrightarrow{\boldsymbol{y}}_{i r_{i}}, \boldsymbol{x}_{i}, \boldsymbol{\phi}_{y}\right)$ in terms of the LDL decomposition of $\Sigma$. Suppose $U$ and $\Lambda$ can be partitioned as $U=\left[\begin{array}{cc}U_{11^{s}} & \mathbf{0} \\ U_{21^{s}} & U_{22^{s}}\end{array}\right]$, and $\Lambda=\operatorname{diag}\left(\Lambda_{1^{s}}, \Lambda_{2^{s}}\right)$ according to the outcomes before and after dropout in pattern $s$. That is, the dimensions of $U_{11^{s}}$, and $\Lambda_{1^{s}}$ are all $s \times s$. Let $L=U^{-1}=\left[\begin{array}{cc}L_{11^{s}} & \mathbf{0} \\ L_{21^{s}} & L_{22^{s}}\end{array}\right]$. Then $\Sigma_{21^{s}} \Sigma_{11^{s}}^{-1}=-U_{22^{s}}^{-1} U_{21^{s}}=-L_{22^{s}} U_{21^{s}}$, and $\Sigma_{2.1^{s}}=\Sigma_{22^{s}}-\Sigma_{21^{s}} \Sigma_{11^{s}}^{-1} \Sigma_{21^{s}}^{\prime}=L_{22^{s}} \Lambda_{2^{s}} L_{22^{s}}^{\prime}$. In pattern $s=0, \Sigma_{2.1^{s}}=\Sigma_{22^{s}}=\Sigma, U_{22^{s}}=U$, and $L_{22^{s}}=L$. Then (5) becomes

$$
\boldsymbol{y}_{i \mathrm{~m}_{2}} \mid \overrightarrow{\boldsymbol{y}}_{i s}, r_{i}=s \sim N\left[\boldsymbol{\eta}_{i m}-L_{22^{s}} U_{21^{s}}\left(\overrightarrow{\boldsymbol{y}}_{i s}-\boldsymbol{\eta}_{i s}\right), L_{22^{s}} \Lambda_{2^{s}} L_{22^{s}}^{\prime}\right]
$$

Below we briefly describe the assumption on missing data in each model. A summary of the assumptions in all models is provided in Table 1. The assumption can be formulated 


\begin{tabular}{|c|c|c|c|c|}
\hline \multirow[b]{2}{*}{ Method } & \multicolumn{2}{|c|}{ Assumption on missing data after dropout } & \multirow[b]{2}{*}{$\begin{array}{c}\text { Conditional mean } \\
\text { of } \boldsymbol{y}_{i \mathrm{~m}_{2}} \text { given }\left(\overrightarrow{\boldsymbol{y}}_{i s}, \boldsymbol{x}_{i}\right)(c, d)\end{array}$} & \multirow[b]{2}{*}{$\begin{array}{c}\text { Mean } \\
\text { Difference }(f)\end{array}$} \\
\hline & $\begin{array}{c}\text { Marginal } \\
\text { mean of } \boldsymbol{y}_{\mathrm{im}_{2}}(a)\end{array}$ & $\begin{array}{c}E\left(y_{i j} \mid \overrightarrow{\boldsymbol{y}}_{i j-1}, \boldsymbol{x}_{i}\right) \\
j>s_{(b)}\end{array}$ & & \\
\hline MMRM (MAR) & $\boldsymbol{\mu}_{i 2^{s}}+\boldsymbol{\delta}_{2^{s}} g$ & $\vartheta_{i j}+\underline{\delta}_{j} g$ & $\boldsymbol{\mu}^{\mathrm{MAR}}=\boldsymbol{\mu}^{\mathrm{CR}}+L_{22^{s}} \underline{\boldsymbol{\delta}}_{2^{s}} g$ & 0 \\
\hline \multicolumn{5}{|c|}{ Controlled Imputation } \\
\hline $\mathrm{J} 2 \mathrm{R}$ & $\boldsymbol{\mu}_{i 2^{s}}$ & - & $\boldsymbol{\mu}^{\mathrm{MAR}}-\boldsymbol{\delta}_{2^{s}} g$ & $-\boldsymbol{\delta}_{2^{s}} g$ \\
\hline CIR & $\boldsymbol{\mu}_{i 2^{s}}+\delta_{s} \mathbf{1}_{p-s} g$ & - & $\boldsymbol{\mu}^{\mathrm{MAR}}+\left(\delta_{s} \mathbf{1}_{p-s}-\boldsymbol{\delta}_{2^{s}}\right) g$ & $\left(\delta_{s} \mathbf{1}_{p-s}-\boldsymbol{\delta}_{2^{s}}\right) g$ \\
\hline $\mathrm{CR}$ & 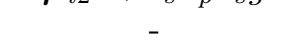 & $\vartheta_{i j}$ & $\boldsymbol{\mu}^{\mathrm{MAR}}-L_{22^{s}} \underline{\boldsymbol{\delta}}_{2^{s}} g$ & $-L_{22^{s}} \underline{\boldsymbol{\delta}}_{2^{s}} g$ \\
\hline ECR & - & $\vartheta_{i j}+(1-\phi) \underline{\delta}_{j} g$ & $\boldsymbol{\mu}^{\mathrm{MAR}}-\phi L_{22^{s}} \underline{\boldsymbol{\delta}}_{2^{s}} g$ & $-\phi L_{22^{s}} \underline{\boldsymbol{\delta}}_{2^{s}} g$ \\
\hline MCR & - & $\vartheta_{i j}+d_{j} \underline{\delta}_{j} g$ & $\boldsymbol{\mu}^{\mathrm{MAR}}-L_{22^{s}} \boldsymbol{\Delta}_{s}^{\mathrm{MCR}} g$ & $-L_{22^{s}} \Delta_{s}^{\mathrm{MCR}} g$ \\
\hline $\begin{array}{l}\text { Delta-adjusted I } \\
\text { conditional } \\
\text { unconditional }\end{array}$ & $\boldsymbol{\mu}_{i 2^{s}}+\left(\boldsymbol{\delta}_{2^{s}}-\boldsymbol{\Delta}_{s}^{u}\right) g$ & $\vartheta_{i j}+\left(\underline{\delta}_{j}-\Delta_{s_{j}}^{c}\right) g$ & $\begin{array}{c}\boldsymbol{\mu}^{\mathrm{MAR}}-L_{22^{s}} \boldsymbol{\Delta}_{s}^{c} g \\
\boldsymbol{\mu}^{\mathrm{MAR}}-\boldsymbol{\Delta}_{s}^{u} g\end{array}$ & $\begin{aligned}- & L_{22_{s}^{s}} \boldsymbol{\Delta}_{s}^{c} g \\
& -\boldsymbol{\Delta}_{s}^{u} g\end{aligned}$ \\
\hline
\end{tabular}

Table 1: Missing data distribution in pattern $s$ for various models: ${ }^{(a)}$ the mean of $\overrightarrow{\boldsymbol{y}}_{i s}$ is $\boldsymbol{\mu}_{i 1^{s}}+\boldsymbol{\delta}_{1^{s}} g$, and the variance of $\boldsymbol{y}_{i}$ is $\Sigma$ in all models. In pattern $s=0, \delta_{s}=0 ;{ }^{(b)} \vartheta_{i j}=\underline{\mu}_{i j}+\sum_{t=1}^{j-1} \beta_{j t} y_{i t}$. The conditional variance of $y_{i j}$ given $\left(\overrightarrow{\boldsymbol{y}}_{i j-1}, \boldsymbol{x}_{i}\right)$ is $\gamma_{j}^{-1}$. (c) the conditional variance of $\boldsymbol{y}_{i \mathrm{~m}_{2}}$ given $\left(\overrightarrow{\boldsymbol{y}}_{i s}, \boldsymbol{x}_{i}\right)$ is $\Sigma_{2.1^{s}}=L_{22^{s}} \Lambda_{2^{s}} L_{22^{s}}^{\prime} ;{ }^{(d)} \boldsymbol{\mu}^{\mathrm{CR}}=L_{22^{s}}\left(\underline{\boldsymbol{\mu}}_{22^{s}}-U_{21^{s}} \overrightarrow{\boldsymbol{y}}_{i s}\right)$. In pattern $s=0, U_{21^{s}} \overrightarrow{\boldsymbol{y}}_{i s}=\mathbf{0} ;{ }^{(f)}$ Difference in mean of $\boldsymbol{y}_{i \mathrm{~m}_{2}}$ between PMM and MMRM.

based either on the marginal distribution of $\boldsymbol{y}_{i \mathrm{~m}_{2}}$ or on the conditional distribution of $y_{i j}$ given the historical outcome. The following notations are used. Suppose subject $i$ is in pattern $r_{i}=s(s<p)$, treatment group $g_{i}=g$. Let $\boldsymbol{e}_{i}$ be a $p-s$ vector of standard normal random variables. Let $\mu_{i j}=\sum_{k=0}^{d} \alpha_{j k} x_{i k}$ denote the mean of subject $i$ at visit $j$ if the subject was on the control treatment, $\boldsymbol{\mu}_{i 1^{s}}=\left(\mu_{i 1}, \ldots, \mu_{i s}\right)^{\prime}$, and $\boldsymbol{\mu}_{i 2^{s}}=\left(\mu_{i, s+1}, \ldots, \mu_{i p}\right)^{\prime}$. Let $\underline{\mu}_{i j}=\mu_{i j}-\sum_{t=1}^{j-1} \beta_{j t} \mu_{i t}=\sum_{k=0}^{d} \underline{\alpha}_{j k} x_{i k}$, and $\underline{\boldsymbol{\mu}}_{i 2^{s}}=\left(\underline{\mu}_{i, s+1}, \ldots, \underline{\mu}_{i p}\right)^{\prime}$. Let $\boldsymbol{\delta}_{1^{s}}=\left(\delta_{1}, \ldots, \delta_{s}\right)^{\prime}$, $\boldsymbol{\delta}_{2^{s}}=\left(\delta_{s+1}, \ldots, \delta_{p}\right)^{\prime}, \underline{\boldsymbol{\delta}}_{1^{s}}=\left(\underline{\delta}_{1}, \ldots, \underline{\delta}_{s}\right)^{\prime}$ and $\underline{\boldsymbol{\delta}}_{2^{s}}=\left(\underline{\delta}_{s+1}, \ldots, \underline{\delta}_{p}\right)^{\prime}$.

\section{a. MMRM (MAR)}

The MMRM assumes dropouts have the same mean response trajectory as completers with identical historical outcome and covariates. By (6),

$\boldsymbol{y}_{i \mathrm{~m}_{2}} \mid \overrightarrow{\boldsymbol{y}}_{i s}, \boldsymbol{x}_{i}, r_{i}=s \sim N\left(\boldsymbol{\mu}^{\mathrm{MAR}}, \Sigma_{2.1^{s}}\right)$, where $U_{21^{s}} \overrightarrow{\boldsymbol{y}}_{i s}=\mathbf{0}$ at $s=0$, and

$\boldsymbol{\mu}^{\mathrm{MAR}}=\boldsymbol{\mu}_{i 2^{s}}+\boldsymbol{\delta}_{2^{s}} g_{i}-L_{22^{s}} U_{21^{s}}\left(\overrightarrow{\boldsymbol{y}}_{i s}-\boldsymbol{\mu}_{i 1^{s}}-\boldsymbol{\delta}_{1^{s}} g_{i}\right)=L_{22^{s}}\left(\underline{\boldsymbol{\mu}}_{i 2^{s}}+\underline{\boldsymbol{\delta}}_{2^{s}} g_{i}-U_{21^{s}} \overrightarrow{\boldsymbol{y}}_{i s}\right)$. Thus

$\boldsymbol{y}_{\mathrm{im}_{2}}$ can be generated in matrix form as

$$
\boldsymbol{y}_{i \mathrm{~m}_{2}}^{\mathrm{MAR}}=L_{22^{s}}\left(\underline{\boldsymbol{\mu}}_{i 2^{s}}+\underline{\boldsymbol{\delta}}_{2^{s}} g_{i}-U_{21^{s}} \overrightarrow{\boldsymbol{y}}_{i s}+\Lambda_{2^{s}}^{1 / 2} \boldsymbol{e}_{i}\right),
$$


or sequentially from the following regression model

$$
y_{i j} \mid \overrightarrow{\boldsymbol{y}}_{i j-1}, \boldsymbol{x}_{i} \sim N\left[\underline{\mu}_{i j}+\sum_{t=1}^{j-1} \beta_{j t} y_{i t}+\underline{\delta}_{j} g_{i}, \gamma_{j}^{-1}\right] \text { for } j>s .
$$

Throughout the paper, the model parameters in the missing data distribution (e.g. (7) and (8) ) are evaluated at the values randomly drawn from their posterior distribution, and in SRI (e.g. (8)) ), the imputed values at previous visits will be used as predictors for imputing the missing values at the next visit.

\section{b. Control-based Imputation}

The control-based imputation assumes that the statistical behavior of active subjects after dropout is similar to that of control subjects, and it reflects the fact that subjects

generally no longer receive the active treatment after dropout. Carpenter et al. (2013) proposed three control-based PMMs: jump to reference (J2R), copy increment in reference (CIR), and CR. These PMMs are suitable for placebo controlled trials and studies where the control treatment consists of a standard-of-care treatment, and subjects discontinued from the active arm tend to switch to standard-of-care (Ratitch et al., 2013). Lu (2014b) considered an extension of the CR approach, which uses a sensitivity parameter to capture the gradual departure from the MAR mechanism. We also suggest a modification of the CR procedure to avoid the possibility that dropouts from the active arm have better mean response trajectory than subjects who remain on the active treatment.

\section{b.1. Jump to Reference (J2R)}

In $\mathrm{J} 2 \mathrm{R}$, once the active subjects cease the treatment, their mean response jumps to that of the control subjects. It essentially assumes that immediately upon withdrawal from the active group, all benefit from the treatment is gone (Mallinckrodt et al., 2013). The mean response is $\boldsymbol{\mu}_{i 1^{s}}+\boldsymbol{\delta}_{1^{s}} g_{i}$ before dropout, and $\boldsymbol{\mu}_{i 2^{s}}$ after dropout. The conditional distribution of $\boldsymbol{y}_{i \mathrm{~m}_{2}}$ given $\left(\overrightarrow{\boldsymbol{y}}_{i s}, \boldsymbol{x}_{i}\right)$ is $N\left(\boldsymbol{\mu}^{\mathrm{J} 2 \mathrm{R}}, \Sigma_{2.1^{s}}\right)$, where $\boldsymbol{\mu}^{\mathrm{J} 2 \mathrm{R}}=\boldsymbol{\mu}_{i 2^{s}}-L_{22^{s}} U_{21^{s}}\left(\overrightarrow{\boldsymbol{y}}_{i s}-\boldsymbol{\mu}_{i 1^{s}}-\boldsymbol{\delta}_{1^{s}} g_{i}\right)=\boldsymbol{\mu}^{\mathrm{MAR}}-\boldsymbol{\delta}_{2^{s}} g_{i}$ for subjects in pattern $s$. Thus $\boldsymbol{y}_{\mathrm{im}_{2}}$ can be generated as

$$
\boldsymbol{y}_{i \mathrm{~m}_{2}}^{\mathrm{J} 2 \mathrm{R}}=\boldsymbol{y}_{i \mathrm{~m}_{2}}^{\mathrm{MAR}}-\boldsymbol{\delta}_{2^{s}} g_{i}
$$




\section{b.2. Copy Increment in Reference (CIR)}

The CIR assumes that the mean profile of active subjects after dropout is parallel to that of control subjects. The mean response is $\boldsymbol{\mu}_{i 1^{s}}+\boldsymbol{\delta}_{1^{s}} g_{i}$ before dropout, and $\boldsymbol{\mu}_{i 2^{s}}+\delta_{s} \mathbf{1}_{p-s} g_{i}$ after dropout, where $\mathbf{1}_{k}$ is a $k \times 1$ vector of ones. The conditional distribution of $\boldsymbol{y}_{i \mathrm{~m}_{2}}$ given $\left(\overrightarrow{\boldsymbol{y}}_{i s}, \boldsymbol{x}_{i}\right)$ is $N\left(\boldsymbol{\mu}^{\mathrm{CIR}}, \Sigma_{2.1^{s}}\right)$, where $\boldsymbol{\mu}^{\mathrm{CIR}}=\boldsymbol{\mu}^{\mathrm{MAR}}+\left(\delta_{s} \mathbf{1}_{p-s}-\boldsymbol{\delta}_{2^{s}}\right) g_{i}$ for subjects in pattern $s$, and $\delta_{s}=0$ at $s=0$ since there is no difference due to treatment in mean baseline response between two arms. We can impute $\boldsymbol{y}_{i \mathrm{~m}_{2}}$ using

$$
\boldsymbol{y}_{i \mathrm{~m}_{2}}^{\mathrm{CIR}}=\boldsymbol{y}_{i \mathrm{~m}_{2}}^{\mathrm{J} \mathrm{R}}+\delta_{s} \mathbf{1}_{p-s} g_{i}=\boldsymbol{y}_{i \mathrm{~m}_{2}}^{\mathrm{MAR}}+\left(\delta_{s} \mathbf{1}_{p-s}-\boldsymbol{\delta}_{2^{s}}\right) g_{i} .
$$

\section{b.3 Copy Reference (CR)}

In CR, the conditional distribution of $\boldsymbol{y}_{i_{2}}$ given $\left(\overrightarrow{\boldsymbol{y}}_{i_{i}}, \boldsymbol{x}_{i}\right)$ among dropouts in the active arm is the same as that of control subjects. That is, $\boldsymbol{y}_{i \mathrm{~m}_{2}} \mid \overrightarrow{\boldsymbol{y}}_{i s}, \boldsymbol{x}_{i}, r_{i}=s \sim N\left(\boldsymbol{\mu}^{\mathrm{CR}}, \Sigma_{2.1^{s}}\right)$, where $\boldsymbol{\mu}^{\mathrm{CR}}=L_{22^{s}}\left(\underline{\boldsymbol{\mu}}_{i 2^{s}}-U_{21^{s}} \overrightarrow{\boldsymbol{y}}_{i s}\right)=\boldsymbol{\mu}^{\mathrm{MAR}}-L_{22^{s}} \underline{\boldsymbol{\delta}}_{2^{s}} g_{i}$. Thus $\boldsymbol{y}_{i \mathrm{~m}_{2}}$ can be imputed as

$$
\boldsymbol{y}_{i \mathrm{~m}_{2}}^{\mathrm{CR}}=\boldsymbol{y}_{i \mathrm{~m}_{2}}^{\mathrm{MAR}}-L_{22^{s}} \underline{\boldsymbol{\delta}}_{2^{s}} g_{i}
$$

The missing data after dropout in both arms can also be imputed sequentially from

$$
y_{i j} \mid \overrightarrow{\boldsymbol{y}}_{i j-1}, \boldsymbol{x}_{i}, r_{i}=s \sim N\left[\underline{\mu}_{i j}+\sum_{t=1}^{j-1} \beta_{j t} y_{i t}, \gamma_{j}^{-1}\right] \text { for } j>s,
$$

which is identical to the zero-dose model of Little and Yau (1996). The method is called copy reference possibly because the missing data distribution is still $N\left(\boldsymbol{\mu}^{\mathrm{CR}}, \Sigma_{2.1^{s}}\right)$ if active subjects are assumed to have the same mean response profiles as the reference (i.e. control) subjects both before and after dropout (Carpenter et al., 2013).

A variant of the CR procedure is implemented in SAS (version 9.4), in which the imputation model is built using only data from the control arm (Ratitch and O'Kelly, 2011; Avele et al., 2014). Although Lu (2014a) showed that two variants of CR performed similarly in a simulation study, the use of only control data may lead to larger random noise in the imputed outcomes if the sample size is small in the control arm.

\section{b.4. Extension of Copy Reference (ECR)}


Lu (2014b) considered an extension of the zero-dose or CR model, which assumes

$$
y_{i j} \mid \overrightarrow{\boldsymbol{y}}_{i j-1}, \boldsymbol{x}_{i}, r_{i}=s \sim N\left[\underline{\mu}_{i j}+(1-\phi) \underline{\delta}_{j} g_{i}+\sum_{t=1}^{j-1} \beta_{j t} y_{i t}, \gamma_{j}^{-1}\right] \text { for } j>s
$$

where $\phi \in[0,1]$ is a pre-specified sensitivity parameter that characterizes the gradual deviation from the MAR mechanism, with $\phi=0$ corresponding to MAR with the full benefit of the active treatment, and $\phi=1$ corresponding to the zero-dose model. The dropout missing data can be imputed from (10) or equivalently from

$$
\boldsymbol{y}_{i \mathrm{~m}_{2}}^{\mathrm{ECR}}=\boldsymbol{y}_{i \mathrm{~m}_{2}}^{\mathrm{MAR}}-\phi L_{22^{s}} \underline{\boldsymbol{\delta}}_{2^{s}} g_{i} .
$$

\section{b.5. A Modification of Copy Reference (MCR)}

Below we illustrate a potential issue with CR using a simple example. Suppose there are only two post-baseline visits $(p=2)$, and the treatment effects are positive $\left(\delta_{1}>0\right.$ and $\delta_{2}>0$ ) at both visits (assuming higher scores represent improvement), but $\underline{\delta}_{2}$ is negative. The mean $y_{i 2}$ is $\sum_{k=0}^{d} \underline{\alpha}_{2 j} x_{i j}+\beta_{21} \mathrm{E}\left(y_{i 1}\right)+\underline{\delta}_{2}$ among active subjects who complete the study, and $\sum_{k=0}^{d} \underline{\alpha}_{2 j} x_{i j}+\beta_{21} \mathrm{E}\left(y_{i 1}\right)$ among active subjects who discontinue after the first visit. In CR, the dropouts have better mean response at visit 2 than completers.

We propose a simple modification of the CR procedure. Let $d_{j}=0$ if $\underline{\hat{\delta}}_{j} \hat{\delta}_{p} \geq 0$, and 1

otherwise, where $\underline{\hat{\delta}}_{j}$ and $\hat{\delta}_{p}$ are the (restricted) maximum likelihood estimates (MLE) from (1). Since the true parameters are unknown, $d_{j}$ 's are determined based on the MLE from (1) prior to the imputation. In MCR, the missing value can be imputed sequentially from

$$
y_{i j} \mid \overrightarrow{\boldsymbol{y}}_{i j-1}, \boldsymbol{x}_{i} \sim N\left[\underline{\mu}_{i j}+d_{j} \underline{\delta}_{j} g_{i}+\sum_{t=1}^{j-1} \beta_{j t} y_{i t}, \gamma_{j}^{-1}\right]
$$

or equivalently in matrix form from

$$
\boldsymbol{y}_{i \mathrm{~m}_{2}}^{\mathrm{MCR}}=\boldsymbol{y}_{i \mathrm{~m}_{2}}^{\mathrm{MAR}}-L_{22^{s}} \boldsymbol{\Delta}_{s}^{\mathrm{MCR}} g_{i},
$$

where $\Delta_{s}^{\mathrm{MCR}}=\left(\left(1-d_{s+1}\right) \underline{\delta}_{s+1}, \ldots,\left(1-d_{p}\right) \underline{\delta}_{p}\right)^{\prime}$. The MCR procedure is identical to the CR procedure if $d_{1}=\ldots=d_{p}=0$. 


\section{c. Delta-adjusted imputation}

In the delta-adjusted PMMs, subjects who discontinue from the active treatment will have their unobserved outcome worse by some pre-specified amount compared with subjects who continue the treatment (Ratitch et al., 2013). The adjustment could be applied in either conditional or unconditional ways.

\section{c.1. Conditional delta-adjusted imputation}

In the conditional approach, the missing data after dropout in pattern $s$ can be imputed sequentially from the following regression model

$$
y_{i j} \mid \overrightarrow{\boldsymbol{y}}_{i j-1}, \boldsymbol{x}_{i} \sim N\left[\underline{\mu}_{i j}+\left(\underline{\delta}_{j}-\Delta_{s_{j}}^{c}\right) g_{i}+\sum_{t=1}^{j-1} \beta_{j t} y_{i t}, \gamma_{j}^{-1}\right]
$$

where $\Delta_{s_{j}}^{c}$ 's are the pre-fixed amount of adjustment at visit $j>s$ for the active subjects in pattern $s$. There are two popular ways to specify $\Delta_{s_{j}}^{c}$ 's (Ratitch et al., 2013;

Mallinckrodt et al., 2013). The adjustment can be applied only once at the first visit after dropout (i.e. $\Delta_{s_{j}}^{c}=\Delta$ when $j=s+1$, and 0 if $j>s+1$ ), or applied to all visits after dropout (i.e. $\Delta_{s_{j}}^{c}=\Delta$ when $s+1 \leq j \leq p$ ). The two adjustment strategies correspond respectively to variant-1 and variant-2 described in Ratitch et al. (2013).

By (13), the conditional distribution of $\boldsymbol{y}_{i \mathrm{~m}_{2}}$ given $\left(\overrightarrow{\boldsymbol{y}}_{i s}, \boldsymbol{x}_{i}\right)$ is $N\left(\boldsymbol{\mu}^{\mathrm{cDEL}}, \Sigma_{2.1^{s}}\right)$, where $\boldsymbol{\Delta}_{s}^{c}=\left(\Delta_{s_{s+1}}^{c}, \ldots, \Delta_{s_{p}}^{c}\right)^{\prime}$ and $\boldsymbol{\mu}^{\mathrm{cDEL}}=L_{22^{s}}\left[\underline{\boldsymbol{\mu}}_{i 2^{s}}+\left(\underline{\boldsymbol{\delta}}_{2^{s}}-\boldsymbol{\Delta}_{s}^{c}\right) g_{i}-U_{21^{s}} \overrightarrow{\boldsymbol{y}}_{i s}\right]$. The missing data after dropout can be imputed from (13) or equivalently from

$$
\boldsymbol{y}_{i \mathrm{~m}_{2}}^{\mathrm{cDEL}}=\boldsymbol{y}_{i \mathrm{~m}_{2}}^{\mathrm{MAR}}-L_{22^{s}} \boldsymbol{\Delta}_{s}^{c} g_{i}
$$

\section{c.2. Unconditional delta-adjusted imputation}

The unconditional approach corresponds to variant-3 of Ratitch et al. (2013). The adjustment is made by simply subtracting a constant from the MAR-based imputation

$$
\boldsymbol{y}_{i \mathrm{~m}_{2}}^{\mathrm{uDEL}}=\boldsymbol{y}_{i \mathrm{~m}_{2}}^{\mathrm{MAR}}-\boldsymbol{\Delta}_{s}^{u} g_{i}
$$

where $\Delta_{s}^{u}=\left(\Delta_{s_{s+1}}^{u}, \ldots, \Delta_{s_{p}}^{u}\right)^{\prime}$ is a vector of pre-specified constants. Unlike the conditional approach in which the adjustment at earlier visits will affect subsequent visits, the 
adjustments at different visits are unrelated in the unconditional approach.

\section{A numerical example}

We analyze an antidepressant clinical trial reported in Mallinckrodt et al. (2013). The Hamilton 17-item rating scale for depression is collected at baseline and weeks 1, 2, 4, 6 . The dataset consists of 84 active subjects, and 88 placebo subjects. The dropout rate is $24 \%(20 / 84)$ in the active arm, and 26\% (23/88) in the placebo arm.

The primary endpoint is the change from baseline in Hamilton depression score, and the explanatory variables are intercept, baseline Hamilton score, and treatment status $(p=4, q=3)$. We analyze the data using both frequentist and Bayesian approaches. In the frequentist approach, the MMRM analysis is fit using SAS PROC MIXED. It includes the treatment $\times$ visit and baseline $\times$ visit interactions as the fixed effects, and an unstructured covariance matrix is used to model the within-patient errors. Our model is different from that used in Mallinckrodt et al. (2013) in that we do not include the investigative site as a covariate. In the Bayesian analysis, we consider three MCMC schemes: the MDA-T algorithm under the prior $f(\boldsymbol{\alpha}, \Sigma) \propto|\Sigma|^{-(p+3) / 2}$ (i.e. $\nu_{0}=2$ ), and the MDA-SAS and FDA-SAS algorithms under Jeffrey's prior $f\left(\boldsymbol{\alpha}_{w}, \Sigma_{w}\right) \propto\left|\Sigma_{w}\right|^{-(p+q) / 2}$. By Lemma 1, the three MCMC schemes yield the same posterior distribution of $(\boldsymbol{\alpha}, \Sigma)$. The latter two analyses are conducted using SAS PROC MI. As shown in Table 2, the treatment effects from the three Bayesian analyses and the likelihood-based analysis are similar since the prior is non-informative.

\begin{tabular}{lcccc}
\hline & & \multicolumn{3}{c}{ Bayesian analysis $^{a}$} \\
\cline { 3 - 5 } Week & Proc Mixed & MDA-T & MDA-SAS & FDA-SAS $^{b}$ \\
\hline 1 & $0.092[-1.256,1.439]$ & $0.092[-1.251,1.436]$ & $0.091[-1.267,1.446]$ & $0.096[-1.245,1.462]$ \\
2 & $-1.403[-3.228,0.422]$ & $-1.404[-3.230,0.434]$ & $-1.402[-3.221,0.430]$ & $-1.395[-3.200,0.440]$ \\
4 & $-2.225[-4.201,-0.248]$ & $-2.219[-4.209,-0.235]$ & $-2.229[-4.213,-0.233]$ & $-2.220[-4.191,-0.251]$ \\
6 & $-2.802[-5.008,-0.596]$ & $-2.793[-5.004,-0.587]$ & $-2.803[-5.016,-0.586]$ & $-2.795[-5.012,-0.557]$ \\
\hline
\end{tabular}

Table 2: Estimated treatment effect [95\% confidence or credible interval] in an antidepressant trial: ${ }^{a}$ posterior mean and quantile-based credible interval are evaluated based on 40,000 MCMC samples collected from every 100-th iteration after a "burn-in" period of 10, 000 iterations; ${ }^{b}$ In FDA-SAS, Jeffrey's prior can be specified with the PRIOR=INPUT= option (adjusted for $1 \mathrm{df}$ in the prior) or PRIOR=JEFFREYS option, and both options produce identical output. 
We also analyze the data using various PMMs, where $m=10,000$ posterior samples are collected from the MDA-SAS algorithm, and the complete datasets are imputed using the strategy in Section 3. Table 3 displays the treatment effect estimate \pm standard error (SE) in MMRM and various PMMs. Under MAR, the MI-based and likelihood-based analyses yield very close results. At week 1, there is no missing data, and the treatment effect estimates are identical in all approaches. In weeks 2, 4, and 6, the PMMs generally yield smaller treatment effect estimate than the MMRM. The MCR and CR produce the same result since $\underline{\hat{\delta}}_{j} \hat{\delta}_{4} \geq 0$ at $j \geq 2$, and $y_{i 1}$ 's are observed for all subjects although $\underline{\hat{\delta}}_{1} \hat{\delta}_{4}<0$. The sample SAS code is provided in the supplementary materials.

\begin{tabular}{|c|c|c|c|c|}
\hline Method & Week 1 & Week 2 & Week 4 & Week 6 \\
\hline \multicolumn{5}{|l|}{ Missing at random } \\
\hline MMRM (ML) & $0.092 \pm 0.683$ & $-1.403 \pm 0.924$ & $-2.225 \pm 1.001$ & $-2.802 \pm 1.116$ \\
\hline MMRM (MI) & $0.092 \pm 0.683$ & $-1.401 \pm 0.925$ & $-2.224 \pm 1.001$ & $-2.806 \pm 1.118$ \\
\hline \multicolumn{5}{|c|}{ Missing not at random } \\
\hline \multicolumn{5}{|c|}{ Control-based imputation } \\
\hline $\mathrm{J} 2 \mathrm{R}$ & $0.092 \pm 0.683$ & $-1.303 \pm 0.927$ & $-1.927 \pm 1.004$ & $-2.126 \pm 1.130$ \\
\hline CIR & $0.092 \pm 0.683$ & $-1.296 \pm 0.926$ & $-2.009 \pm 1.001$ & $-2.451 \pm 1.109$ \\
\hline CR & $0.092 \pm 0.683$ & $-1.297 \pm 0.926$ & $-1.975 \pm 1.001$ & $-2.372 \pm 1.109$ \\
\hline $\mathrm{ECR}: \phi=0.5$ & $0.092 \pm 0.683$ & $-1.349 \pm 0.925$ & $-2.100 \pm 0.999$ & $-2.589 \pm 1.109$ \\
\hline MCR & $0.092 \pm 0.683$ & $-1.297 \pm 0.926$ & $-1.975 \pm 1.001$ & $-2.372 \pm 1.109$ \\
\hline \multicolumn{5}{|c|}{ Conditional delta-adjusted imputation } \\
\hline $\boldsymbol{\Delta}_{s}^{c}=(-4,0, \ldots, 0)^{\prime}$ & $0.092 \pm 0.683$ & $-1.122 \pm 0.938$ & $-1.800 \pm 1.009$ & $-2.020 \pm 1.141$ \\
\hline $\boldsymbol{\Delta}_{s}^{c}=(-2, \ldots,-2)^{\prime}$ & $0.092 \pm 0.683$ & $-1.261 \pm 0.930$ & $-1.873 \pm 1.010$ & $-2.047 \pm 1.139$ \\
\hline \multicolumn{5}{|c|}{ Unconditional delta-adjusted imputation } \\
\hline $\boldsymbol{\Delta}_{s}^{u}=(-3, \ldots,-3)^{\prime}$ & $0.092 \pm 0.683$ & $-1.192 \pm 0.934$ & $-1.826 \pm 1.010$ & $-2.082 \pm 1.136$ \\
\hline
\end{tabular}

Table 3: Estimated treatment effect \pm SE by visit in MMRM and various PMMs. PMMs are implemented via MI based on $m=10,000$ imputations. Posterior samples are collected from every 100-th iteration after a "burn-in" period of 10,000 iterations using the MDA-SAS algorithm.

\section{Comparison with several existing methods}

This section reviews several existing imputation methods. These methods generally produce similar results to the algorithm proposed in section 3 if they make the same assumptions on the observed data distribution (i.e. same observed data likelihood), and the number of imputations is large enough to stabilize the result. The difference in results is usually small, and it arises because of the use of different priors, and imputation 
variability due to the use of a finite number of imputations.

The main advantages of the proposed algorithm are (1) it sufficiently uses the existing functions of SAS PROC MI, and the SAS code is simpler and easier to maintain and/or modify, and (2) the algorithm reaches stationarity quickly particularly if one chooses the MDA-SAS approach for imputation in MMRM. It is also more convenient to use the proposed method to compare different PMMs in sensitivity analysis. One can simply save the imputed datasets for MMRM, and the posterior samples of $\boldsymbol{\phi}_{y}$, and then use the method described in Section 3 to derive the imputed dataset for each PMM.

\subsection{Sequential regression imputation (SRI)}

The SRI approach (Little and Yau, 1996; Ratitch et al., 2013; Lu, 2014a) is a popular imputation method for monotone data. It can be viewed as a special case of the MDA-T algorithm. Under monotone missingness, the MDA-T algorithm involves only the P-step, and reaches stationary in one step. When $A=\mathbf{0}, M=\mathbf{0}$ and $p(\boldsymbol{\alpha}, \Sigma) \propto|\Sigma|^{-\left(\nu_{0}+p+1\right) / 2}$, the posterior distribution (19) in Appendix A.1 can be expressed as (Tang, 2016)

$$
\gamma_{j} \mid Y_{\mathrm{m}_{1}}, Y_{o} \sim \chi_{n_{j}+\nu_{0}+j-q-p}^{2} / \hat{S}_{j} \text { and } \boldsymbol{\theta}_{j} \mid \gamma_{j}, Y_{\mathrm{m}_{1}}, Y_{o} \sim N\left[\hat{\boldsymbol{\theta}}_{j},\left(\gamma_{j} \sum_{i=1}^{n_{j}} \boldsymbol{z}_{i, j-1}^{\prime} \boldsymbol{z}_{i, j-1}\right)^{-1}\right]
$$

where $\hat{\boldsymbol{\theta}}_{j}=\left(\sum_{i=1}^{n_{j}} \boldsymbol{z}_{i, j-1}^{\prime} \boldsymbol{z}_{i, j-1}\right)^{-1}\left(\sum_{i=1}^{n_{j}} \boldsymbol{z}_{i, j-1}^{\prime} y_{i j}\right)$ and $\hat{S}_{j}=\sum_{i=1}^{n_{j}}\left(y_{i j}-\boldsymbol{z}_{i, j-1}^{\prime} \hat{\boldsymbol{\theta}}_{j}\right)^{2}$. The missing data after dropout can be sequentially imputed from (8), (9), (10), (11), (13) respectively for MMRM, CR, ECR, MCR, and conditional delta-adjusted PMM.

In the literature, the SRI approach is mainly applied to models with simple conditional distribution of $y_{i j}$ given $\overrightarrow{\boldsymbol{y}}_{i j-1}$. But it is also suitable for complicate PMMs such as J2R and CIR using the method described in Section 3 ,

The SRI approach is available in SAS (version 9.4) PROC MI for imputations under MAR, and the conditional delta-adjusted imputations (using MNAR and MONOTONE statements). In SAS, the posterior distribution of $\gamma_{j} \sim \chi_{n_{j}-q-(j-1)}^{2} / \hat{S}_{j}$ is slightly different from (16) due to the use of a different prior. 


\subsection{Ratitch et al. (2013) approach}

Ratitch et al. (2013) described a procedure for data with intermittent missing values. It firstly uses Schafer (1997) MDA algorithm implemented in SAS to impute the intermittent missing data $\left(Y_{\mathrm{m} 1}\right) m$ times. The SRI approach is then used to impute $Y_{\mathrm{m} 2}$ for each imputed monotone dataset. The sampling schemes are different in Ratitch et al. (2013) method and the proposed algorithm (assume MDA algorithm is used)

- Ratitch et al scheme: Iterate between $\boldsymbol{\phi}_{x}, \boldsymbol{\phi}_{y} \mid X, Y_{o}, Y_{\mathrm{m} 1}$ and $Y_{\mathrm{m} 1} \mid \boldsymbol{\phi}_{x}, \boldsymbol{\phi}_{y}, X, Y_{o}$ until convergence. Sample $\boldsymbol{\phi}_{y}^{*} \mid X, Y_{\mathrm{m} 1}, Y_{o}$, and $Y_{\mathrm{m} 2} \mid \boldsymbol{\phi}_{y}^{*}, X, Y_{\mathrm{m} 1}, Y_{o}$ after convergence.

- Proposed scheme: Iterate between $\boldsymbol{\phi}_{x}, \boldsymbol{\phi}_{y} \mid X, Y_{o}, Y_{\mathrm{m} 1}$ and $Y_{\mathrm{m} 1} \mid \boldsymbol{\phi}_{x}, \boldsymbol{\phi}_{y}, X, Y_{o}$ until convergence. Sample $Y_{\mathrm{m} 2} \mid \phi_{y}, X, Y_{\mathrm{m} 1}, Y_{o}$ after convergence.

Compared to Ratitch et al. (2013) method, the proposed scheme avoids one additional step in sampling $\boldsymbol{\phi}_{y}^{*}$, and it can save computational time particularly if the number of imputations $m$ is large, or if one wants to compare different PMMs in sensitivity analysis.

Similarly to SRI, Ratitch et al. (2013) method is developed for the CR and conditional delta-adjusted imputation, but it can be modified to handle J2R and CIR.

\subsection{Macro based on SAS Proc MCMC}

Mallinckrodt et al. (2013) developed a SAS package for missing data imputation in PMMs, and it is freely available at http://www.missingdata.org.uk. The package is based on SAS PROC MCMC, in which $(\boldsymbol{\alpha}, \Sigma)$ is sampled using Metropolis-type algorithms. The convergence of the Metropolis-type algorithm can be slow particularly if the dimension of parameter space is large (e.g. when the number of post-baseline visits $p$ is large). The real clinical data are usually monotone or approximately monotone, and the MDA algorithm generally converges much more quickly with smaller autocorrelation between posterior samples than the Metropolis-type algorithm. For the antidepressant trial analyzed in Section 4, the MDA algorithm converges within 100 iterations, and the lag-1 autocorrelation is close to 0 for all model parameters (Tang, 2016). Furthermore, our SAS code is much simpler, and runs much faster than Mallinckrodt et al. (2013) 
macro for the same number of MCMC iterations. But Mallinckrodt et al. (2013) macro can handle more complex MMRM (e.g. covariance matrix heterogeneity).

\section{Discussion}

The PMMs have been widely used as sensitivity analysis of longitudinal outcomes with non-ignorable missing data. We describe a novel approach for missing value imputations in the MMRM, delta-adjusted PMMs and control-based PMMs. The imputed values in PMMs can be obtained from that under MAR by subtracting the mean difference in their posterior predictive distributions, which is a function of the posterior samples of the MMRM model parameters $\phi_{y}$. We have focused on the control-based and delta-adjustment PMMs. However, the imputation algorithm works for any PMMs that assume the same observed data distribution as MMRM.

For CR, it is possible to impute missing data in an alternative way using SAS PROC MI. One may firstly impute the intermittent missing data $\boldsymbol{y}_{i_{1}}$ 's under MAR. The dropout missing data $\boldsymbol{y}_{\mathrm{im}_{2}}$ 's can be imputed by setting the treatment status as placebo for dropouts in both arms.

In (1), the covariance matrix is assumed to be homogeneous across all subjects, and there is a covariate $\times$ visit interaction for each covariate, so that the MCMC sampling can be easily implemented using SAS PROC MI. For more complex MMRMs, one may use Tang (2016) MDA algorithm or Mallinckrodt et al. (2013) SAS macro.

There is considerable debate regarding the appropriateness of using Rubin's variance estimator when the data imputation and analysis models are uncongenial (Meng, 1994). In control-based PMMs, Rubin's variance estimator overestimates the variance of the estimated treatment effect (Lu, 2014a; Avele et al., 2014). Intuitively, this is because the data are imputed on an as-treated basis (Little and Yau, 1996), but analyzed under the ITT principle. In a companion paper, we show that Rubin's variance estimator is approximately unbiased in delta-adjusted PMMs, and this provides theoretical support for the use of delta-adjusted PMMs as MNAR sensitivity analysis in clinical trials.

Imputations under MNAR based on the fully conditional specification (FCS) method are implemented in SAS (version 9.4). The FCS approach involves specifying the 
conditional distribution for each incomplete variable given the other variables, and iterating the imputations on a variable-by-variable basis until convergence (van Buuren, 2007). It can be challenging to find the stationary distribution of the missing data in the FCS approach under MNAR, but it is different from that in the MCMC method since the intermittent missing data are MAR in the MCMC algorithms (see Section 3), and MNAR in the FCS approach (this can easily be seen in the special case where all outcomes are observed except few subjects miss the first visit). Further research shall be done to understand the FCS imputation under MNAR.

\section{Acknowledgments}

We would like to thank the associate editor and referees for their constructive comments that greatly help to improve the quality of the article.

\section{SUPPLEMENTARY MATERIAL}

Sample SAS code for the analysis of the antidepressant trial is provided in the supplementary materials. The raw dataset is freely available at www.missingdata.org.uk.

\section{A Appendix}

\section{A.1 MDA and FDA algorithms for MMRM}

Tang (2015, 2016) developed the MDA algorithm (called MDA-T) for MMRM. Compared to Schafer (1997) MDA algorithm for incomplete multivariate normal data, the new MDA method allows the use of both non-informative and informative priors, and greatly reduces the amount of matrix inversion in imputing $\left(\boldsymbol{y}_{i \mathrm{~m}_{1}}, \boldsymbol{y}_{\mathrm{im}_{2}}\right)$ 's from their posterior predictive distribution. In addition, the new MDA algorithm can handle more complex assumptions on the mean and covariance of $\boldsymbol{y}_{i}$ 's.

In the prior, $\left(\boldsymbol{\theta}_{j}, \gamma_{j}\right)$ 's follow independent normal-gamma distributions (Tang, 2015)

$$
f\left(\boldsymbol{\theta}_{j}, \gamma_{j}\right) \propto \gamma_{j}^{\frac{\nu_{0}+2 j+r-p-3}{2}} \exp \left[-\frac{\gamma_{j}}{2} \tilde{\boldsymbol{\theta}}_{j}^{\prime}\left(\tilde{A}_{j}+B_{j}\right) \tilde{\boldsymbol{\theta}}_{j}\right]
$$


where $\tilde{\boldsymbol{\theta}}_{j}=\left(-\boldsymbol{\theta}_{j}^{\prime}, 1\right)^{\prime}, \tilde{A}_{j}$ is the leading $(q+j) \times(q+j)$ submatrix of the $(q+p) \times(q+p)$ matrix $\tilde{A}=\left[\begin{array}{ll}\mathbf{0} & \mathbf{0} \\ \mathbf{0} & A\end{array}\right], I_{q}$ is the $q \times q$ identity matrix, $\ddot{\boldsymbol{\alpha}}_{j}=\left(I_{q}, \boldsymbol{\alpha}_{1}, \ldots, \boldsymbol{\alpha}_{j}\right)$ and $B_{j}=\ddot{\boldsymbol{\alpha}}_{j}^{\prime} M \ddot{\boldsymbol{\alpha}}_{j}$.

The likelihood function for the augmented monotone data is given by

$$
\prod_{i=1}^{n} f\left(\boldsymbol{y}_{i r_{i}} \mid \boldsymbol{x}_{i}, \boldsymbol{\phi}_{y}\right)=\prod_{j=1}^{p} \gamma_{j}^{\frac{n_{j}}{2}} \exp \left[-\frac{\gamma_{j}}{2} \tilde{\boldsymbol{\theta}}_{j}^{\prime}\left(\sum_{i=1}^{n_{j}} \boldsymbol{z}_{i j} \boldsymbol{z}_{i j}^{\prime}\right) \tilde{\boldsymbol{\theta}}_{j}\right]
$$

Combining (17) and (18) yields the posterior distribution for $\left(\boldsymbol{\theta}_{j}, \gamma_{j}\right)$ 's, which is normal-gamma, and can be generated using the methods of Tang (2016, 2015)

$$
f\left(\boldsymbol{\theta}_{j}, \gamma_{j} \mid Y_{\mathrm{m}_{1}}, Y_{o}, X\right) \propto \gamma_{j}^{\frac{n_{j}+\nu_{0}+2 j+r-p-3}{2}} \exp \left[-\frac{\gamma_{j}}{2} \tilde{\boldsymbol{\theta}}_{j}^{\prime} D_{j} \tilde{\boldsymbol{\theta}}_{j}\right]
$$

where $D_{j}=\tilde{A}_{j}+B_{j}+\sum_{i=1}^{n_{j}} \boldsymbol{z}_{i j} \boldsymbol{z}_{i j}^{\prime}$. Tang (2016) derived the posterior distribution of $\boldsymbol{y}_{i \mathrm{~m}_{1}}$

$$
f\left(\boldsymbol{y}_{i \mathrm{~m}_{1}} \mid \boldsymbol{y}_{i o}, \phi_{y}\right) \propto \prod_{j=h}^{r_{i}} \exp \left[-\frac{\gamma_{j}\left(\tilde{U}_{j m}^{\prime} \boldsymbol{y}_{i \mathrm{~m}_{1}}-e_{i j}\right)^{2}}{2}\right] \propto N\left(\hat{\mu}_{y_{\mathrm{m}_{1}}}, \hat{V}_{y_{\mathrm{m} 1}}\right)
$$

where $h\left(h<r_{i}\right)$ is the index of the first missing observation for subject $i,\left(\tilde{U}_{j m}, \tilde{U}_{j o}\right)$ is a partition of the $\left(r_{i}-h+1\right) \times 1$ vector $\tilde{U}_{j}=\left(-\beta_{j h}, \ldots,-\beta_{j, j-1}, 1,0, \ldots, 0\right)^{\prime}$ according to the missing $\left(\boldsymbol{y}_{i \mathrm{~m}_{1}}\right)$ and observed $\left(\boldsymbol{y}_{i o_{h}}\right)$ parts of $\left(y_{i h}, \ldots, y_{i r_{i}}\right)^{\prime}$ for $j \geq h$, $e_{i j}=\underline{\boldsymbol{\alpha}}_{j}^{\prime} \boldsymbol{x}_{i}+\sum_{t=1}^{h-1} \beta_{j t} y_{i t}-\tilde{U}_{j o}^{\prime} \boldsymbol{y}_{i o_{h}}\left(\tilde{U}_{j m}^{\prime} \boldsymbol{y}_{i \mathrm{~m}_{1}}-e_{i j}=y_{i j}-\underline{\boldsymbol{\alpha}}_{j}^{\prime} \boldsymbol{x}_{i}-\sum_{t=1}^{j-1} \beta_{j t} y_{i t}\right)$, $\hat{V}_{y_{\mathrm{m} 1}}=\left(\sum_{j=h}^{r_{i}} \gamma_{j} \tilde{U}_{j m} \tilde{U}_{j m}^{\prime}\right)^{-1}$ and $\hat{\mu}_{y_{\mathrm{m}_{1}}}=\hat{V}_{y_{\mathrm{m} 1}} \sum_{j=h}^{r_{i}} \gamma_{j} e_{i j} \tilde{U}_{j m}$.

The MDA-T algorithm repeats the following I- and P- steps until convergence

P: Draw $\left(\boldsymbol{\theta}_{j}, \gamma_{j}\right)$ from the posterior distribution (19) for $j=1, \ldots, p$;

I: Impute $\boldsymbol{y}_{i \mathrm{~m}_{1}}$ 's from (20) for subjects with intermittent missing data.

Similarly, a FDA algorithm can be developed on basis of the posterior distribution of $\left(\boldsymbol{\theta}_{j}, \gamma_{j}\right)$ given the augmented full data $Y_{f}=\left\{Y_{o}, Y_{\mathrm{m}_{1}}, Y_{\mathrm{m}_{2}}\right\}$

$$
f\left(\boldsymbol{\theta}_{j}, \gamma_{j} \mid Y_{f}, X\right) \propto \gamma_{j}^{\frac{n+\nu_{0}+2 j+r-p-3}{2}} \exp \left[-\frac{\gamma_{j}}{2} \tilde{\boldsymbol{\theta}}_{j}^{\prime}\left(\tilde{A}_{j}+B_{j}+\sum_{i=1}^{n} \boldsymbol{z}_{i j} \boldsymbol{z}_{i j}^{\prime}\right) \tilde{\boldsymbol{\theta}}_{j}\right]
$$


The algorithm (called FDA-T) repeats the following steps until convergence.

P: Draw $\left(\boldsymbol{\theta}_{j}, \gamma_{j}\right)$ from its posterior distribution (21) for $j=1, \ldots, p$;

I: Impute $\boldsymbol{y}_{i \mathrm{~m}_{1}}$ 's from (20), and $\boldsymbol{y}_{\mathrm{m}_{2}}$ 's from $f\left(\boldsymbol{y}_{\mathrm{m}_{2}} \mid \overrightarrow{\boldsymbol{y}}_{i_{i}}, \boldsymbol{x}_{i}, \boldsymbol{\phi}_{y}\right)$.

\section{A.2 Proof of Lemma 1}

Proof Let $\bar{M}=\left[\begin{array}{cc}0 & \mathbf{0}^{\prime} \\ \mathbf{0} & M^{*}\end{array}\right]$ and $\underline{M}=\left[\begin{array}{cc}m_{11} & M_{12} \\ M_{21} & \underline{M}^{*}\end{array}\right]$, where $\underline{M}^{*}=M_{21} M_{12} / m_{11}$ if $m_{11}>0$, and $\underline{M}^{*}=\mathbf{0}$ if $m_{11}=0$. Then $\bar{M}+\underline{M}=M$. Let $\tilde{j}=q-1+j$. By Lemma 2 of Tang (2015), in the prior, $\left(\boldsymbol{\theta}_{j}^{*}, \gamma_{j}^{*}\right)^{\prime}$ 's are independent, and the distribution of $\left(\boldsymbol{\theta}_{\tilde{j}}^{*}, \gamma_{\tilde{j}}^{*}\right)$ is

$$
f\left(\boldsymbol{\theta}_{\tilde{j}}^{*}, \gamma_{\tilde{j}}^{*}\right) \propto \gamma_{\tilde{j}}^{* \frac{\nu_{w}+2(q-1+j)+r_{w}-(q-1+p)-3}{2}} \exp \left[-\frac{\gamma_{\tilde{j}}^{*}}{2} \tilde{\boldsymbol{\theta}}_{\tilde{j}}^{*^{\prime}}\left(\tilde{A}_{w_{j}}+B_{w_{j}}\right) \tilde{\boldsymbol{\theta}}_{\tilde{j}}^{*}\right]
$$

where $\tilde{A}_{w}=\left[\begin{array}{cc}0 & \mathbf{0}_{\tilde{p}}^{\prime} \\ \mathbf{0}_{\tilde{p}} & A_{w}\end{array}\right]=\left[\begin{array}{cc}\bar{M} & \bar{M} \boldsymbol{\alpha}_{0}^{\prime} \\ \boldsymbol{\alpha}_{0} \bar{M} & A+\boldsymbol{\alpha}_{0} \bar{M} \boldsymbol{\alpha}_{0}^{\prime}\end{array}\right], \tilde{\boldsymbol{\theta}}_{\tilde{j}}^{*}=\left(-\boldsymbol{\theta}_{\tilde{j}}^{*^{\prime}}, 1\right)^{\prime}, u_{w}=\left(1, \boldsymbol{\alpha}_{w 0}^{\prime}\right)^{\prime}$,

$B_{w}=m_{11} u_{w}^{\prime} u_{w}, \tilde{A}_{w_{j}}$ and $B_{w_{j}}$ denote respectively the leading $(q+j) \times(q+j)$ submatrix of $A_{w}$ and $B_{w}, r_{w}=1$ if $m_{11}>0$, and $r_{w}=0$ if $m_{11}=0$. A little algebra shows that $\tilde{A}_{w_{j}}=\ddot{\boldsymbol{\alpha}}_{j}^{\prime} \bar{M} \ddot{\boldsymbol{\alpha}}_{j}+\tilde{A}_{j}, B_{w_{j}}=\ddot{\boldsymbol{\alpha}}_{j}^{\prime} \underline{M} \ddot{\boldsymbol{\alpha}}_{j}$ and $\tilde{A}_{w_{j}}+B_{w_{j}}=B_{j}+\tilde{A}_{j}$. Thus

$$
f\left(\boldsymbol{\theta}_{\tilde{j}}^{*}, \gamma_{\tilde{j}}^{*}\right) \propto \gamma_{\tilde{j}}^{*} \frac{\nu_{0}+2 j+r-p-3}{2} \exp \left[-\frac{\gamma_{\tilde{j}}^{*}}{2} \tilde{\boldsymbol{\theta}}_{\tilde{j}}^{*^{\prime}}\left(\tilde{A}_{j}+B_{j}\right) \tilde{\boldsymbol{\theta}}_{\tilde{j}}^{*}\right],
$$

and $\left(\boldsymbol{\theta}_{\tilde{j}}^{*}, \gamma_{\tilde{j}}^{*}\right)$ has the same prior distribution as $\left(\boldsymbol{\theta}_{j}, \gamma_{j}\right)$ defined in (17).

Since $\phi_{x}$ and $\phi_{y}$ are independent in the prior, and the likelihood function can be factored as the product of $\prod_{i=1}^{n} f\left(\tilde{\boldsymbol{x}}_{i} \mid \boldsymbol{\phi}_{x}\right)$ and $\prod_{i=1}^{n} f\left(\boldsymbol{y}_{i o} \mid \boldsymbol{x}_{i}, \boldsymbol{\phi}_{y}\right), \boldsymbol{\phi}_{x}$ and $\boldsymbol{\phi}_{y}$ are independent in the posterior distribution. The posterior distribution of $\phi_{y}$ is $f\left(\boldsymbol{\phi}_{y} \mid Y_{o}, X\right) \propto \prod_{i=1}^{n} f\left(\boldsymbol{y}_{i o} \mid \boldsymbol{x}_{i}, \boldsymbol{\phi}_{y}\right) \prod_{j=1}^{p} f\left(\boldsymbol{\theta}_{\tilde{j}}^{*}, \gamma_{\tilde{j}}^{*}\right)$, which is identical to that from any valid MCMC algorithm for (1).

Since $\left(\boldsymbol{\alpha}_{x}, \Sigma_{x x}\right)$ is a function of $\boldsymbol{\phi}_{x}$, and $(\boldsymbol{\alpha}, \Sigma)$ is a function of $\boldsymbol{\phi}_{y},\left(\boldsymbol{\alpha}_{x}, \Sigma_{x x}\right)$ and $(\boldsymbol{\alpha}, \Sigma)$ are independent in both the prior and posterior distributions, and the posterior distribution of $(\boldsymbol{\alpha}, \Sigma)$ is identical that from any valid MCMC algorithm for (1). 


\section{A.3 MCMC algorithms in SAS}

The MDA-SAS algorithm repeats the following steps until the algorithm converges.

P1: Sample $\left(\boldsymbol{\theta}_{j}^{*}, \gamma_{j}^{*}\right)^{\prime}$ s from $f\left(\boldsymbol{\theta}_{j}^{*}, \gamma_{j}^{*} \mid X\right)$ for $j=1, \ldots, q-1$.

P2: Draw $\left(\boldsymbol{\theta}_{q-1+j}^{*}, \gamma_{q-1+j}^{*}\right)$ 's from $f\left(\boldsymbol{\theta}_{q-1+j}^{*}, \gamma_{q-1+j}^{*} \mid Y_{\mathrm{m}_{1}}, Y_{o}, X\right)$ for $j=1, \ldots, p$.

I: Impute missing data $\boldsymbol{y}_{i_{1}}$ 's from $f\left(\boldsymbol{y}_{i \mathrm{~m}_{1}} \mid \boldsymbol{y}_{i o}, \boldsymbol{x}_{i}, \boldsymbol{\phi}_{y}\right)$.

The P2 and I steps in the MDA-SAS algorithm are equivalent to the $\mathrm{P}$ and I steps in the MDA-T algorithm, and are unrelated to the P1-step. This explains the equivalence of the MDA-T and MDA-SAS algorithms.

The FDA-SAS algorithm iterates between the following steps until convergence.

I: Impute missing data $\boldsymbol{y}_{i \mathrm{~m}_{1}}$ 's and $\boldsymbol{y}_{i \mathrm{~m}_{2}}$ 's;

P: Sample $\left(\boldsymbol{\alpha}_{w}, \Sigma_{w}\right)$ from their posterior distribution

$$
\begin{aligned}
\Sigma_{w} \mid Y_{f}, X & \sim \mathcal{W}^{-1}\left(\sum_{i=1}^{n}\left(\boldsymbol{w}_{i}-\overline{\boldsymbol{w}}\right)^{\otimes 2}+A_{w}+\frac{n m_{11}}{n+m_{11}}\left(\overline{\boldsymbol{w}}-\boldsymbol{\alpha}_{w 0}\right)^{\otimes 2}, f\right), \\
\boldsymbol{\alpha}_{w} \mid \Sigma_{w}, Y_{f}, X & \sim N\left(\frac{1}{n+m_{11}}\left(n \overline{\boldsymbol{w}}+m_{11} \boldsymbol{\alpha}_{w 0}\right), \frac{1}{n+m_{11}} \Sigma_{w}\right)
\end{aligned}
$$

where $A^{\otimes 2}=A A^{\prime}, \overline{\boldsymbol{w}}=n^{-1} \sum_{i=1}^{n} \boldsymbol{w}_{i}, f=n+\nu_{w}-1$ if $m_{11}=0$ and $f=n+\nu_{w}$ if $m_{11}>0$. The P-steps of the FDA-SAS and FDA-T algorithms seem quite different. If $\left(\boldsymbol{\alpha}_{w}, \Sigma_{w}\right)$ is distributed as $(23),\left(\boldsymbol{\theta}_{q-1+j}^{*}, \gamma_{q-1+j}^{*}\right)$ has in fact the same posterior distribution as $\left(\boldsymbol{\theta}_{j}, \gamma_{j}\right)$ defined (21) , and this can be proved using the fact that the random Wishart matrix can be expressed as a function of independent normal-gamma random variables (Tang, 2015).

Schafer (1997) obtained (23) under the normal-inverse-Wishart prior (i.e. $m_{11} \neq 0$ ).

The proof will be similar when $m_{11}=0$, at which (23) reduces to

$$
\begin{aligned}
\Sigma_{w} \mid Y_{f}, X & \sim \mathcal{W}^{-1}\left(\sum_{i=1}^{n}\left(\boldsymbol{w}_{i}-\overline{\boldsymbol{w}}\right)^{\otimes 2}+A_{w}, n+\nu_{w}-1\right) \\
\boldsymbol{\alpha}_{w} \mid \Sigma_{w}, Y_{f}, X & \sim N\left(\overline{\boldsymbol{w}}, n^{-1} \Sigma_{w}\right) .
\end{aligned}
$$


SAS (version 9.4) PROC MI uses wrong degrees of freedom (df) $f=n+\nu_{w}$ when $m_{11}=0$ (i.e. $f\left(\boldsymbol{\alpha}_{w} \mid \Sigma_{w}\right) \propto$ constant), and one specifies the prior with the PRIOR $=\mathrm{INPUT}=$ option. One may reduce the $\mathrm{df}$ in the prior by 1 (i.e. from $\nu_{w}$ to $\nu_{w}-1$ ) in

order to get the right posterior distribution. The df is right in SAS PROC MI if $m_{11}>0$, or if one specifies Jeffrey's prior using the PRIOR=Jeffreys option $\left(m_{11}=0\right)$.

\section{References}

Ayele, B. T., I. Lipkovich, G. Molenberghs, and C. H. Mallinckrodt (2014). A multiple-imputation-based approach to sensitivity analyses and effectiveness assessments in longitudinal clinical trials. Journal of Biopharmaceutical Statistics 24(2), $211-28$.

Carpenter, J., J. Roger, and M. Kenward (2013). Analysis of longitudinal trials with protocol deviation: a framework for relevant, accessible assumptions, and inference via multiple imputation. Journal of Biopharmaceutical Statistics 23, 1352 - 71.

CHMP (2010). EMA Guideline on Missing data in Confirmatory Clinical Trials (EMA/CPMP/EWP/1776/99). London: CHAMP.

ICH E9 (1999). Statistical principles for clinical trials: Ich harmonized tripartite guideline. Statistics in Medicine 18, 1905 - 42.

Little, R. and L. Yau (1996). Intent-to-treat analysis for longitudinal studies with drop-outs. Biometrics 52, $1324-33$.

Liu, J. S. (1994). The collapsed Gibbs sampler in Bayesian computations with applications to a gene regulation problem. Journal of the American Statistical Association 89, $958-66$.

Lu, K. (2014a). An analytic method for the placebo-based pattern-mixture model. Statistics in Medicine 33, 1134-45.

Lu, K. (2014b). An extension of the placebo-based pattern-mixture model. Pharmaceutical Statistics 13, 103-9. 
Mallinckrodt, C., J. Roger, C. Chuang-stein, G. Molenberghs, P. W. Lane, M. Okelly, B. Ratitch, L. Xu, S. Gilbert, D. V. Mehrotrak, R. Wolfinger, and H. Thijs (2013). Missing data: Turning guidance into action. Statistics in Biopharmaceutical Research 5, $369-82$.

Meng, X. (1994). Multiple-imputation inference with uncongenial sources of input. Statistical Science 9,538-73.

National Research Council (2010). The prevention and treatment of missing data in clinical trials. The National Academies Press: Washington, DC.

Phillips, A., J. Abellan-Andres, A. Soren, F. Bretz, C. Fletcher, L. France, A. Garrett, R. Harris, M. Kjaer, O. Keene, D. Morgan, and M. O. andJames Roger (2016). Estimands: discussion points from the psi estimands and sensitivity expert group. Pharmaceutical Statistics.

Ratitch, B. and M. O'Kelly (2011). Implementation of pattern-mixture models using standard SAS/STAT procedures. In in Proceedings of PharmaSUG 2011 (Pharmaceutical Industry SAS Users Group), SP04, Nashville.

Ratitch, B., M. O'Kelly, and R. Tosiello (2013). Missing data in clinical trials: from clinical assumptions to statistical analysis using pattern mixture models. Pharmaceutical Statistics 12, $337-47$.

Rubin, D. (1987). Multiple Imputation for Nonresponse in Surveys. Wiley: New York.

Schafer, J. L. (1997). Analysis of Incomplete Multivariate Data. Chapman Hall, London.

Siddiqui, O., J. H. M. Hung, and R. O’Neill (2009). MMRM vs. LOCF: A comprehensive comparison based on simulation study and 25 NDA datasets. Journal of Biopharmaceutical Statistics 19, 227-46.

Tang, Y. (2015). An efficient monotone data augmentation algorithm for Bayesian analysis of incomplete longitudinal data. Statistics and Probability Letters 104, 146 52. 
Tang, Y. (2016). An efficient monotone data augmentation algorithm for multiple imputation in a class of pattern mixture models. Journal of Biopharmaceutical Statistics.

van Buuren, S. (2007). Multiple imputation of discrete and continuous data by fully conditional specification. Statistical Methods in Medical Research 16, 219 - 42. 